\title{
On stopping Fock-space processes
}

\author{
Alexander C. R. Belton \\ Department of Mathematics and Statistics \\ Lancaster University, United Kingdom \\ a.belton@lancaster.ac.uk Tuesday 31st July 2018
}

\begin{abstract}
We consider the theory of stopping bounded processes within the framework of HudsonParthasarathy quantum stochastic calculus, for both identity and vacuum adaptedness. This provides significant new insight into Coquio's method of stopping (J. Funct. Anal. 238:149$180,2006)$. Vacuum adaptedness is required to express certain quantum stochastic representations, and many results, including the proof of the optional-sampling theorem, take a more natural form.
\end{abstract}

Key words: quantum stopping time; quantum stop time; quantum stochastic calculus; regular quantum semimartingale; regular $\Omega$-semimartingale.

MSC 2010: 81S25 (primary); 46L53, 60G40 (secondary).

\section{Introduction}

The extension of the notion of stopping time from classical to non-commutative probability is straightforward, with the earliest definition in the literature due to Hudson [20]. The idea was developed in the setting of the Clifford probability gauge space by Barnett and Lyons [9], and for abstract filtered von Neumann algebras by Barnett and Thakrar 10, 11, and Barnett and Wilde [12, 13]; see also [31, where Sauvageot initiated a programme to solve a $C^{*}$-algebraic version of the Dirichlet problem, and recent work by Łuczak [26].

In the more concrete setting of Hudson-Parthasarathy quantum stochastic calculus [23], an extensive theory was developed by Parthasarathy and Sinha [30. In particular, they showed that, given a quantum stopping time $S$, the Boson Fock space $\mathcal{F}=\mathcal{F}_{+}\left(L^{2}\left(\mathbb{R}_{+} ; \mathrm{k}\right)\right)$ has the factorisation $\mathcal{F}_{S)} \otimes \mathcal{F}_{[S}$, where the spaces $\mathcal{F}_{S)}$ and $\mathcal{F}_{[S}$ are pre- $S$ and post- $S$ spaces; this provides a form of the strong Markov property that generalises Hudson's result [20]. Further contributions in this setting have been made by Meyer [28, Accardi and Sinha [1, Attal and Sinha [8], Sinha [32], Attal and Coquio [4, Coquio [19] and Hudson [21, 22]. Quantum stopping times are applied to the CCR flow and its cocycles in [16] and [17, building on work of Applebaum [2]. Parthasarathy and his collaborators developed the theory around another quantum-probabilistic version of the Dirichlet problem: see [6], [18, 7] and [29].

Here, we investigate stopping in parallel for both identity-adapted and vacuum-adapted versions of quantum stochastic calculus; the latter variant was introduced in [14 and further developed in [15]. By developing the two forms of the theory in parallel, we provide insights which illuminate Coquio's constructions in [19] and allow us to extend her results. Certain integrals, which 
seem somewhat mysterious from the identity-adapted viewpoint, can be seen to be products due to switching forms of adaptedness. Furthermore, the vacuum-adapted theory is seen to have superior properties, which correspond more naturally to the classical situation. For simplicity and clarity of exposition, we restrict our attention to processes composed of bounded operators.

In the Fock-space context, a quantum stopping time $S$ is a projection-valued measure on the extended half line $[0, \infty]$, such that $S([0, \infty])=I$, the identity operator on $\mathcal{F}$, and $t \mapsto S([0, t])$ is an identity-adapted process, i.e., $S([0, t]) \in B\left(\mathcal{F}_{t)}\right) \otimes I_{[t}$ for all $t \geqslant 0$. (Here the Boson Fock space $\mathcal{F}$ is identified with $\mathcal{F}_{t)} \otimes \mathcal{F}_{(t}$; this is the familiar deterministic version of the Parthasarathy-Sinha factorisation.) Section 2 presents this definition, derives some basic results from it and sets out some classical and non-commutative examples.

The primary object associated with a quantum stopping time $S$ is its time projection $E_{S}$, and this is introduced in Section 3, if $S$ is deterministic, so that $S(\{t\})=I$ for some $t$, then $E_{S}$ is the orthogonal projection $E_{t}=I_{t)} \otimes P_{[t}^{\Omega}$ onto $\mathcal{F}_{t} \otimes \varepsilon(0)$, where here $\varepsilon(0)$ is the vacuum vector in $\mathcal{F}_{[t}$. If $\mathcal{F}$ is identified with the $L^{2}$ space of a standard Brownian motion with filtration $\mathcal{A}=\left(\mathcal{A}_{t}\right)_{t \in \mathbb{R}_{+}}$and $S$ corresponds to a classical $\mathcal{A}$-stopping time $\tau$ then $E_{S}$ becomes the conditional expectation with respect to the stopping time $\sigma$-algebra $\mathcal{A}_{\tau}$. The time projection $E_{S}$ has a quantum stochastic representation (Theorem 3.6):

$$
E_{S}=I+\int_{0}^{\infty} I_{\mathrm{k}} \otimes S((s, \infty]) E_{s} \mathrm{~d} \Lambda_{s}
$$

The integrand is a vacuum-adapted process, which shows that this form of adaptedness appears naturally when considering quantum stopping times.

If $S$ is a quantum stopping time and $X=\left(X_{t}\right)_{t \in \mathbb{R}_{+}}$is a process (i.e., a family of operators on $\mathcal{F}$ satisfying suitable measurability and adaptedness conditions) then there are three natural approaches to stopping $X$ at $S$ : these are

$$
\begin{aligned}
& \qquad S \cdot X:=S(\{0\}) X_{0}+\lim _{\pi} \sum_{j=0}^{\infty} S\left(\left(\pi_{j-1}, \pi_{j}\right]\right) X_{\pi_{j}}, \\
& \qquad X \cdot S:=X_{0} S(\{0\})+\lim _{\pi} \sum_{j=0}^{\infty} X_{\pi_{j}} S\left(\left(\pi_{j-1}, \pi_{j}\right]\right) \\
& \text { and } \quad S \cdot X \cdot S:=S(\{0\}) X_{0} S(\{0\})+\lim _{\pi} \sum_{j=0}^{\infty} S\left(\left(\pi_{j-1}, \pi_{j}\right]\right) X_{\pi_{j}} S\left(\left(\pi_{j-1}, \pi_{j}\right]\right), \quad \text { (doubl) } \\
& \text { assuming these limits, taken over partitions of } \mathbb{R}_{+} \text {, exist in an appropriate sense. (To establish } \\
& \text { convergence is often difficult, or apparently impossible in general.) The time projection } E_{S} \text { is } \\
& \text { the result of stopping the vacuum-adapted process }\left(E_{t}\right)_{t \in \mathbb{R}_{+}} \text {in any of these three senses. Each } \\
& \text { of the expressions yields the same orthogonal projection, and convergence holds in the strong } \\
& \text { operator topology, since these projections form an decreasing family as the partition is refined: } \\
& \text { see Theorem } 3.3 \text { below. }
\end{aligned}
$$


In the vacuum-adapted setting, the value at time $t$ of a martingale $M$ closed by the operator $M_{\infty}$ is simply $E_{t} M_{\infty} E_{t}$; see Section 5 for the definitions of martingales in this context. Thus it is natural to define $M_{\breve{S}}$, the value of the martingale $M$ stopped in a vacuum-adapted manner at $S$, to be $E_{S} M_{\infty} E_{S}$; it is easy to see that this equals $S \cdot M \cdot S$, the result of double stopping $M$ at $S$. The issue of convergence becomes that of the existence of $E_{S}$, which is long established, and this definition has various good properties: see Sections 4 and 7 below. In particular, the optional-sampling theorem, Theorem 7.4, holds, and is a immediate consequence of the identity $E_{S} \wedge E_{T}=E_{S \wedge T}$, which is true for any two quantum stopping times $S$ and $T$ (Theorem 3.11).

In [19], Coquio puts forward a method of stopping for identity-adapted processes which is not obviously one of the forms given above. She begins by working with discrete stopping times, i.e., those with finite support: if $T$ is a quantum stopping time with support $\left\{t_{1}<\cdots<t_{n}\right\} \subseteq \mathbb{R}_{+}$ and $X$ is a process then

$$
X_{\widehat{T}}:=\sum_{i, j=1}^{n} \widehat{\pi}\left(E_{t_{i}}\right)_{t_{i} \vee t_{j}} T\left(\left\{t_{i}\right\}\right) \widehat{\pi}\left(X_{t_{i} \vee t_{j}}\right)_{t_{i} \vee t_{j}} T\left(\left\{t_{j}\right\}\right) \widehat{\pi}\left(E_{t_{j}}\right)_{t_{i} \vee t_{j}}
$$

is the result of applying identity-adapted stopping to $X$ at $T$, where $\widehat{\pi}$ is the projection onto the space of identity-adapted processes. For example, the operator $\widehat{\pi}\left(E_{s}\right)_{t}=I_{s)} \otimes P_{[s, t)}^{\Omega} \otimes I_{[t}$ maps $\mathcal{F}_{[s, t)}$ onto the vacuum subspace and acts as the identity on $\mathcal{F}_{s)}$ and $\mathcal{F}_{[t}$, with $\mathcal{F}$ identified with $\mathcal{F}_{s)} \otimes \mathcal{F}_{[s, t)} \otimes \mathcal{F}_{[t}$. (This notation is explained further in Section 5,) In Section 6 the vacuum-adapted version of this definition is introduced and various consequences are derived; in particular, Lemma 6.9 shows that, for a closed martingale, it agrees with the natural definition of $M_{\breve{T}}$ described above. In particular, Coquio's method is seen to be a form of double stopping, at least for a large class of processes.

Working from the definition (1.2), Coquio obtains an integral formula for the stopped process $M_{\widehat{T}}$ when $M$ is a closed martingale (see Theorem 6.14), and uses this to extend the definition of $M_{\widehat{T}}$ to arbitrary stopping times. The key step in this result, Theorem 7.5 below, is to show that, given any quantum stopping time $S$ and any $Z \in B(\mathcal{F})$, the sum

$$
E_{S} Z E_{S}+\int_{0}^{\infty} I_{\mathrm{k}} \otimes S([0, s]) \widehat{\pi}\left(E_{S} Z E_{S}\right)_{s} S([0, s]) \mathrm{d} \Lambda_{s}
$$

extends to a bounded operator $Z_{\widehat{S}}$; we provide a somewhat shorter version of Coquio's proof in Section 17. It follows that the difference between stopping a closed martingale $M$ at an arbitrary quantum stopping time $S$ in the identity-adapted and vacuum-adapted senses is given by a gauge integral:

$$
M_{\widehat{S}}-M_{\breve{S}}=\int_{0}^{\infty} I_{\mathrm{k}} \otimes S([0, s]) \widehat{\pi}\left(M_{\breve{S}}\right)_{s} S([0, s]) \mathrm{d} \Lambda_{s} ;
$$

in particular, the integral in Coquio's definition of $M_{\widehat{S}}$ can be seen as an artifact produced by working with identity, rather than vacuum, adaptedness.

In Section 8, vacuum-adapted stopping for FV processes and for semimartingales is extended from discrete to arbitrary times. An FV process $Y$ is of the form $Y_{t}=\int_{0}^{t} H_{s} \mathrm{~d} s$ for some integrand process $H$, and a semimartingale is the sum of a martingale and an FV process. 
Given sufficient regularity, a semimartingale may be written as the sum of four quantum stochastic integrals. Such a process is called a regular quantum semimartingale if identity adapted, and a regular $\Omega$-semimartingale if vacuum adapted. The integral formula (1.1) for $E_{S}$ is used in Section 9 to show that the class of regular $\Omega$-semimartingales is closed under vacuumadapted stopping (Theorem 9.2); Coquio has obtained the analogous result for regular quantum semimartingales [19, Proposition 3.16].

In the final Section 10, it is shown that the projection onto the future space may be stopping in the three different senses, and that a natural relationship holds between these stopped operators. Each has a vacuum-adapted quantum stochastic representation.

An appendix, Section A, is included to gather the necessary results on quantum stochastic integration.

\subsection{Acknowledgement}

The author is grateful for the referee's comments on a previous version of this work.

\subsection{Notation and conventions}

The term "increasing" applies in the weak sense. Hilbert spaces have complex scalar fields; inner products are linear in the second argument. The indicator function of the set $A$ is denoted by $1_{A}$. The complement of an orthogonal projection $P$ is denoted by $P^{\perp}$. The set of natural numbers $\mathbb{N}=\{1,2,3, \ldots\}$, the set of non-negative integers $\mathbb{Z}_{+}=\mathbb{N} \cup\{0\}$ and the set of non-negative real numbers $\mathbb{R}_{+}=[0, \infty)$. The von Neumann algebra of bounded operators on a Hilbert space $\mathbf{H}$ is denoted by $B(\mathrm{H})$. Given a real number $x$, its ceiling $\lceil x\rceil$ is the smallest integer at least as great as $x$.

\section{Quantum stopping times}

Notation 2.1. Let $\mathcal{F}_{A}=\mathcal{F}_{+}\left(L^{2}(A ; \mathrm{k})\right)$ denote Boson Fock space over $L^{2}(A ; \mathrm{k})$, where $A$ is a subinterval of $\mathbb{R}_{+}$and $\mathrm{k}$ is a complex Hilbert space. For brevity, let $\mathcal{F}:=\mathcal{F}_{\mathbb{R}_{+}}, \mathcal{F}_{t)}:=\mathcal{F}_{[0, t)}$ for all $t \in(0, \infty)$ and $\mathcal{F}_{[t}:=\mathcal{F}_{[t, \infty)}$ for all $t \in \mathbb{R}_{+}$, with similar abbreviations $I, I_{t)}$ and $I_{[t}$ for the identity operators on these spaces.

The set of exponential vectors $\left\{\varepsilon(f): f \in L^{2}\left(\mathbb{R}_{+} ; \mathbf{k}\right)\right\}$ is total in $\mathcal{F}$ and linearly independent, with $\langle\varepsilon(f), \varepsilon(g)\rangle=\exp \langle f, g\rangle$ for all $f, g \in L^{2}\left(\mathbb{R}_{+} ; \mathrm{k}\right)$; we let $\mathcal{E}$ denote the linear span of this set.

Definition 2.2 ([30, Section 3]). A spectral measure on $\mathcal{B}[0, \infty]$, the Borel $\sigma$-algebra on the extended half-line $[0, \infty]:=\mathbb{R}_{+} \cup\{\infty\}$, is a map

$$
S: \mathcal{B}[0, \infty] \rightarrow B(\mathrm{H})
$$

where $\mathrm{H}$ is a complex Hilbert space, such that 
(i) the operator $S(A)$ is an orthogonal projection for all $A \in \mathcal{B}[0, \infty]$;

(ii) the mapping $\mathcal{B}[0, \infty] \rightarrow \mathbb{C} ; A \mapsto\langle x, S(A) y\rangle$ is a complex measure for all $x, y \in \mathcal{F}$;

(iii) the total measure $S([0, \infty])=I$.

The spectral measure $S$ is a quantum stopping time if $\mathrm{H}=\mathcal{F}$ and $S$ is identity adapted in the following sense:

(iv) the operator $S(\{0\}) \in \mathbb{C} I$ and $S([0, t]) \in B\left(\mathcal{F}_{t)}\right) \otimes I_{[t}$ for all $t \in(0, \infty)$.

Remark 2.3 (Cf. [9, Definitions 3.1]). A spectral measure on $\mathcal{B}[0, \infty]$ may also be defined to be an increasing family of orthogonal projections $\left(S_{t}\right)_{t \in[0, \infty]}$ in $B(\mathrm{H})$ such that $S_{\infty}=I$. The equivalence of these definitions is ensured by the spectral theorem for unbounded self-adjoint operators.

Proposition 2.4. Let $S$ be a spectral measure on $\mathcal{B}[0, \infty]$, and let $A$ and $B$ be arbitrary elements of $\mathcal{B}[0, \infty]$.

(i) The operator $S(\emptyset)=0$.

(ii) If $A \subseteq B$ then $S(A) S(B)=S(A)=S(B) S(A)$.

(iii) If $A$ and $B$ are disjoint then $S(A \cup B)=S(A)+S(B)$ and $S(A) S(B)=0$.

(iv) In general, $S(A) S(B)=S(A \cap B)=S(B) S(A)$.

Proof. This is a simple exercise.

Lemma 2.5. If $S: \mathcal{B}[0, \infty] \rightarrow B(\mathrm{H})$ is a spectral measure then $t \mapsto S([0, t]) x$ is right continuous on $\mathbb{R}_{+}$, so strongly measurable, for all $x \in \mathrm{H}$.

Proof. Let $x \in \mathrm{H}$ and note that if $s, t \in \mathbb{R}_{+}$with $s<t$ then

$$
\|S([0, t]) x-S([0, s]) x\|^{2}=\|S((s, t]) x\|^{2}=\langle x, S((s, t]) x\rangle .
$$

Thus if $t_{n} \rightarrow s+$ then, since $\cap_{n}\left(s, t_{n}\right]=\emptyset$, so $S\left(\left[0, t_{n}\right]\right) x \rightarrow S([0, s]) x$. Hence setting

$$
f_{n}: \mathbb{R}_{+} \rightarrow \mathrm{H} ; t \mapsto 1_{[0, n]}(t) S\left(\left[0,2^{-n}\left\lceil 2^{n} t\right\rceil\right]\right) x \quad \text { for all } n \in \mathbb{N}
$$

gives a sequence of simple functions converging pointwise to $t \mapsto S([0, t]) x$.

The following result will be applied without comment.

Corollary 2.6. Let $S: \mathcal{B}[0, \infty] \rightarrow B(\mathrm{H})$ be a spectral measure.

(i) If $f: \mathbb{R}_{+} \rightarrow \mathrm{H}$ is strongly measurable then so is $t \mapsto S([0, t]) f(t)$.

(ii) If $F: \mathbb{R}_{+} \rightarrow B(\mathrm{H})$ is pointwise strongly measurable, so that $t \mapsto F(t) x$ is strongly measurable for all $x \in \mathrm{H}$, then so is $t \mapsto F(t) S([0, t])$. 
Proof. For all $n \geqslant 1$, let

$S_{n}(t):=1_{[0, n]}(t) S\left(\left[0,2^{-n}\left\lceil 2^{n} t\right\rceil\right]\right)= \begin{cases}S(\{0\}) & \text { if } t=0, \\ S\left(\left[0, j 2^{-n}\right]\right) & \text { if } t \in\left((j-1) 2^{-n}, j 2^{-n}\right]\left(j=1, \ldots, n 2^{n}\right), \\ 0 & \text { if } t \in(n, \infty) .\end{cases}$

(i) If $N \subseteq \mathbb{R}_{+}$is a Lebesgue-null set and $\left(f_{n}\right)_{n \geqslant 1}$ is a sequence of simple functions such that $\left\|f_{n}(t)-f(t)\right\| \rightarrow 0$ for all $t \in \mathbb{R}_{+} \backslash N$ then $t \mapsto S_{n}(t) f_{n}(t)$ is a simple function and

$$
\left\|S_{n}(t) f_{n}(t)-S([0, t]) f(t)\right\| \leqslant\left\|f_{n}(t)-f(t)\right\|+\left\|\left(S_{n}(t)-S([0, t])\right) f(t)\right\| \rightarrow 0
$$

if $t \in \mathbb{R}_{+} \backslash N$.

(ii) Let $x \in \mathrm{H}$ and note that $t \mapsto F(t) S_{n}(t) x$ is the sum of finitely many strongly measurable functions, so is thus itself strongly measurable. Since $F(t) S_{n}(t) x \rightarrow F(t) S([0, t]) x$ for all $t \in \mathbb{R}_{+}$, the claim follows.

Example 2.7. For any $t \in[0, \infty]$, setting

$$
t: \mathcal{B}[0, \infty] \rightarrow B(\mathcal{F}) ; A \mapsto \begin{cases}0 & \text { if } t \notin A \\ I & \text { if } t \in A\end{cases}
$$

defines a quantum stopping time which corresponds to the deterministic time $t$.

Example 2.8. Let $B=\left(B_{t}\right)_{t \in \mathbb{R}_{+}}$be a standard Brownian motion and use the Wiener-ItôSegal transform to identify the Fock space $\mathcal{F}$, where $\mathrm{k}=\mathbb{C}$, with the space $L^{2}(\Omega, \mathcal{A}, \mathbb{P})$. If $\tau$ is a classical stopping time for $B$ then

$$
S: \mathcal{B}[0, \infty] \rightarrow B(\mathcal{F}) ; \quad A \mapsto 1_{\{\tau \in A\}}
$$

is a quantum stopping time, where the function $1_{\{\tau \in A\}}$ acts by multiplication on $L^{2}(\Omega, \mathcal{A}, \mathbb{P})$. The same applies with $B$ replaced by any classical process with the chaotic representation property, e.g., the classical or monotone Poisson processes, or Azéma's martingale: see [3, Section II.1].

Example 2.9 (4, pp.508-510]). Let $\nu=\left(\nu_{t}\right)_{t \in \mathbb{R}_{+}}$be a standard Poisson process with intensity 1 and unit jumps on the probability space $(\Omega, \mathcal{A}, \mathbb{P})$, where $\mathcal{A}$ is complete and generated by $\nu$; for all $n \in \mathbb{Z}_{+}$, let

$$
\tau_{n}:=\inf \left\{t \in \mathbb{R}_{+}: \nu_{t}=n\right\}
$$

be the $n$th jump time. The fact that

$$
\int_{0}^{t} \phi_{s}(\omega) \mathrm{d} \nu_{s}(\omega)=\sum_{k=1}^{\nu_{t}(\omega)} \phi_{\tau_{k}(\omega)}(\omega) \quad \text { for all } \omega \in \Omega
$$

where $\phi$ is any process, together with the identity $\left\{\nu_{t} \geqslant n\right\}=\left\{\tau_{n} \leqslant t\right\}$, implies that

$$
\int_{0}^{t}\left(1_{\left\{\tau_{n-1}<s\right\}}(\omega)-1_{\left\{\tau_{n}<s\right\}}(\omega)\right) \mathrm{d} \nu_{s}(\omega)=\int_{0}^{t} 1_{\left(\tau_{n-1}(\omega), \tau_{n}(\omega)\right]}(s) \mathrm{d} \nu_{s}(\omega)=1_{\left\{\tau_{n} \leqslant t\right\}}(\omega)
$$


for all $\omega \in \Omega, n \in \mathbb{N}$ and $t \in \mathbb{R}_{+}$. Since $\left(\nu_{t}-t\right)_{t \in \mathbb{R}_{+}}$is a normal martingale and $\tau_{m}$ has the gamma distribution with mean and variance $m$, it holds that

$$
\mathbb{E}\left[\left(\int_{0}^{t} 1_{\left\{\tau_{m}=s\right\}} \mathrm{d}\left(\nu_{s}-s\right)\right)^{2}\right]=\mathbb{E}\left[\int_{0}^{t} 1_{\left\{\tau_{m}=s\right\}} \mathrm{d} s\right]=\int_{0}^{t} \mathbb{P}\left(\tau_{m}=s\right) \mathrm{d} s=0
$$

and therefore, as elements of $L^{2}(\Omega, \mathcal{A}, \mathbb{P})$,

$$
1_{\left\{\tau_{n} \leqslant t\right\}}=\int_{0}^{t}\left(1_{\left\{\tau_{n-1} \leqslant s\right\}}-1_{\left\{\tau_{n} \leqslant s\right\}}\right) \mathrm{d} \nu_{s} \quad \text { for all } t \in \mathbb{R}_{+} .
$$

With $\mathrm{k}=\mathbb{C}$, let $N_{t}=\Lambda_{t}+A_{t}+A_{t}^{\dagger}+t I$ for all $t \in \mathbb{R}_{+}$, so that $N$ is the usual quantum stochastic representation of the Poisson process $\nu$ : there exists an isometric isomorphism $U_{P}$ : $L^{2}(\Omega, \mathcal{A}, \mathbb{P}) \rightarrow \mathcal{F}$ such that $U_{P}^{*} N_{t} U_{P}$ is essentially self adjoint, with closure corresponding to multiplication by $\nu_{t}$, for all $t \in \mathbb{R}_{+}$, and $U_{P} 1=\varepsilon(0)$ [23, Theorems 6.1-2]. It follows from Lemma $\$$.10 that

$$
\int_{0}^{t} T_{n}([0, s]) \mathrm{d} N_{s}=U_{P} \int_{0}^{t} 1_{\left\{\tau_{n} \leqslant s\right\}} \mathrm{d} \nu_{s} U_{P}^{*} \quad \text { on } \mathcal{E}
$$

for all $t \in \mathbb{R}_{+}$, where the quantum stopping time $T_{n}$ is defined by setting

$$
T_{n}(A):=U_{P} 1_{\left\{\tau_{n} \in A\right\}} U_{P}^{*} \quad \text { for all } n \in \mathbb{Z}_{+} \text {and } A \in \mathcal{B}[0, \infty],
$$

as in Example 2.8, and the stochastic integral on the right-hand side of (2.2) acts by multiplication on $L^{2}(\Omega, \mathcal{A}, \mathbb{P})$. In particular, $T_{n}$ satisfies the quantum stochastic differential equation

$$
T_{n}([0, t])=\int_{0}^{t}\left(T_{n-1}([0, s])-T_{n}([0, s])\right) \mathrm{d} N_{s} \quad \text { for all } t \in \mathbb{R}_{+} \text {and } n \in \mathbb{N} .
$$

Definition 2.10 ([9, Definitions 3.1], [30, Section 3]). A partial order is defined on quantum stopping times in the following manner: if $S$ and $T$ are quantum stopping times then $S \leqslant T$ if and only if $S([0, t]) \geqslant T([0, t])$ for all $t \in[0, \infty]$. The definition agrees with the classical ordering in Examples 2.8 and 2.9 ; in the latter, $T_{m} \leqslant T_{n}$ for all $m, n \in \mathbb{Z}_{+}$such that $m \leqslant n$.

Theorem 2.11. If $S$ is a quantum stopping time and $s \in[0, \infty]$ then $S \wedge s$ is a quantum stopping time, where

$$
(S \wedge s)([0, t])=\left\{\begin{array}{cl}
S([0, t]) & \text { if } t<s, \\
I & \text { if } t \geqslant s
\end{array}\right.
$$

for all $t \in[0, \infty]$. Furthermore, if $s, t \in[0, \infty]$ with $s \leqslant t$ then

$$
S \wedge s \leqslant S \wedge t \leqslant S, \quad S \wedge s \leqslant s \quad \text { and } \quad(S \wedge t) \wedge s=S \wedge s .
$$

Proof. This is a straightforward exercise. 
Remark 2.12 (Cf. [10, Lemma 2.3],[30, Proposition 3.1]). Quantum stopping times $S \wedge T$ and $S \vee T$ are defined for any pair of quantum stopping times $S$ and $T$ by setting

$$
\begin{aligned}
(S \wedge T)([0, t]) & =S([0, t]) \vee T([0, t]) \\
\text { and } \quad(S \vee T)([0, t]) & =S([0, t]) \wedge T([0, t]) \quad \text { for all } t \in[0, \infty] .
\end{aligned}
$$

It is straightforward to verify that $t \mapsto(S \wedge T)([0, t])$ and $t \mapsto(S \vee T)([0, t])$ are increasing. Furthermore, $S \wedge T \leqslant S \leqslant S \vee T$ and $S \wedge T \leqslant T \leqslant S \vee T$.

If $S([0, t])$ commutes with $T([0, t])$ then

$$
\begin{aligned}
(S \wedge T)([0, t]) & =S([0, t])+T([0, t])-S([0, t]) T([0, t]) \\
\text { and } \quad(S \vee T)([0, t]) & =S([0, t]) T([0, t]),
\end{aligned}
$$

for all $t \in[0, \infty]$.

\section{Time projections}

Definition 3.1. Let $E_{0} \in B(\mathcal{F})$ be the orthogonal projection onto $\mathbb{C} \varepsilon(0)$, let $E_{t} \in B(\mathcal{F})$ be the orthogonal projection onto $\mathcal{F}_{t} \otimes \varepsilon\left(\left.0\right|_{[t, \infty)}\right)$, considered as a subspace of $\mathcal{F}$, for all $t \in(0, \infty)$ and let $E_{\infty}:=I$. Then

$$
E_{t} \varepsilon(f)=\varepsilon\left(1_{[0, t)} f\right) \quad \text { for all } t \in[0, \infty] \text { and } f \in L^{2}\left(\mathbb{R}_{+} ; \mathbf{k}\right) .
$$

Given a quantum stopping time $S$, the time projection

$$
E_{S}=\int_{[0, \infty]} S(\mathrm{~d} s) E_{s+}=\int_{[0, \infty]} E_{s+} S(\mathrm{~d} s)
$$

where these integrals are strongly convergent limits of Riemann sums: see Theorem 3.3, Note that left, right and double stopping $E=\left(E_{t}\right)_{t \in[0, \infty]}$ at $S$ produce the same result, as observed in the Introduction.

Definition 3.2. A strictly increasing sequence $\pi=\left(\pi_{j}\right)_{j \in \mathbb{Z}_{+}}$with $\pi_{0}=0$ and $\lim _{j \rightarrow \infty} \pi_{j}=\infty$ is said to be a partition of $\mathbb{R}_{+}$. A partition $\pi^{\prime}$ is a refinement of $\pi$ if $\pi$ is a subsequence of $\pi^{\prime}$.

The following theorem dates back at least as far as [11, Theorem 2.3].

Theorem 3.3. Let $S$ be a quantum stopping time and let $\pi$ be a partition of $\mathbb{R}_{+}$. The series

$$
E_{S}^{\pi}:=S(\{0\}) E_{0}+\sum_{j=1}^{\infty} S\left(\left(\pi_{j-1}, \pi_{j}\right]\right) E_{\pi_{j}}+S(\{\infty\})
$$

converges in the strong operator topology to an orthogonal projection. If $\pi^{\prime}$ is a refinement of $\pi$ then $E_{S}^{\pi^{\prime}} \leqslant E_{S}^{\pi}$, so $E_{S}:=$ st. $\lim _{\pi} E_{S}^{\pi}$ exists and is an orthogonal projection such that $E_{S} \leqslant E_{S}^{\pi}$ for all $\pi$. Furthermore, it holds that $E_{S} S([0, t])=S([0, t]) E_{S}$ for all $t \in[0, \infty]$. 
Proof. Note first that if $s, t \in \mathbb{R}_{+}$are such that $s<t$ then

$$
S((s, t])=S([0, t])-S([0, s]) \in B\left(\mathcal{F}_{t)}\right) \otimes I_{[t} \quad \text { and } \quad E_{t} \in I_{t)} \otimes B\left(\mathcal{F}_{[t}\right) .
$$

Hence if $m, n \in \mathbb{Z}_{+}$are such that $m \leqslant n$ then

$$
\begin{aligned}
\left\|\sum_{j=m+1}^{n} S\left(\left(\pi_{j-1}, \pi_{j}\right]\right) E_{\pi_{j}} x\right\|^{2} & =\sum_{j=m}^{n}\left\|S\left(\left(\pi_{j-1}, \pi_{j}\right]\right) E_{\pi_{j}} x\right\|^{2} \\
& =\sum_{j=m+1}^{n}\left\|E_{\pi_{j}} S\left(\left(\pi_{j-1}, \pi_{j}\right]\right) x\right\|^{2} \\
& \leqslant \sum_{j=m+1}^{n}\left\|S\left(\left(\pi_{j-1}, \pi_{j}\right]\right) x\right\|^{2} \\
& \leqslant\left\|S\left(\left(\pi_{m}, \infty\right)\right) x\right\|^{2}
\end{aligned}
$$

for all $x \in \mathcal{F}$, which gives the first claim; that $E_{S}^{\pi}$ is an orthogonal projection is immediately verified. For the second, note first that if $s, t \in \mathbb{R}_{+}$are such that $0<s \leqslant t$ then

$$
\left(E_{t}-E_{s}\right)^{2}=E_{t}^{2}+E_{s}^{2}-2 E_{s}=E_{t}-E_{s} \in I_{s)} \otimes B\left(\mathcal{F}_{[s}\right) .
$$

For all $j \in \mathbb{Z}_{+}$, let $k_{j} \geqslant j$ be such that $\pi_{k_{j}}^{\prime}=\pi_{j}$ and let $l_{j} \geqslant 1$ be such that $\pi_{k_{j}+l_{j}}^{\prime}=\pi_{j+1}$. Then

$$
\left\langle x,\left(E_{S}^{\pi}-E_{S}^{\pi^{\prime}}\right) x\right\rangle=\sum_{j=0}^{\infty} \sum_{l=1}^{l_{j}}\left\langle x, S\left(\left(\pi_{k_{j}+l-1}^{\prime}, \pi_{k_{j}+l}^{\prime}\right]\right)\left(E_{\pi_{k_{j}+l_{j}}^{\prime}}-E_{\pi_{k_{j}+l}^{\prime}}\right) x\right\rangle \geqslant 0
$$

for all $x \in \mathcal{F}$, as required. The final claim is readily verified.

Remark 3.4. If the quantum stopping time $S$ corresponds to the deterministic time $t \in[0, \infty]$, so that $S(\{t\})=I$, then $E_{S}=E_{t}$.

Example 3.5 ([9, Remark 3.6]). Let $M$ be a normal martingale with the chaotic-representation property, such as a standard Brownian motion, and identity the Fock space $\mathcal{F}$ with $L^{2}(\Omega, \mathcal{A}, \mathbb{P})$, as in Examples 2.8 and 2.9. If $\tau$ is a classical stopping time for $M$ then

$$
E_{T} \xi=\mathbb{E}\left[\xi \mid \mathcal{A}_{\tau}\right] \quad \text { for all } \xi \in L^{2}(\Omega, \mathcal{A}, \mathbb{P}),
$$

where $T$ is the quantum stopping time corresponding to $\tau$, as in Example 2.8, and $\mathcal{A}_{\tau}$ is the $\sigma$ algebra at the stopping time $\tau$. To see that (3.2) holds, note first that, in this interpretation of Fock space, the exponential vector $\varepsilon(f)$ is a stochastic exponential and satisfies the stochastic differential equation [27, Section II.2]

$$
\varepsilon(f)=1+\int_{0}^{\infty} f(t) \varepsilon\left(1_{[0, t)} f\right) \mathrm{d} M_{t} \quad \text { for all } f \in L^{2}\left(\mathbb{R}_{+} ; \mathrm{k}\right),
$$


therefore

$$
\mathbb{E}\left[\varepsilon(f) \mid \mathcal{A}_{t}\right]=1+\int_{0}^{t} f(s) \varepsilon\left(1_{[0, s)} f\right) \mathrm{d} M_{s}=\varepsilon\left(1_{[0, t)} f\right)=E_{t} \varepsilon(f)
$$

for all $t \in[0, \infty]$. Hence if $\tau$ takes values in the set $\left\{t_{1}<\cdots<t_{n}\right\}$ then

$$
\mathbb{E}\left[\xi \mid \mathcal{A}_{\tau}\right]=\sum_{i=1}^{n} 1_{\left\{\tau=t_{i}\right\}} \mathbb{E}\left[\xi \mid \mathcal{A}_{t_{i}}\right]=\sum_{i=1}^{n} 1_{\left\{\tau=t_{i}\right\}} E_{t_{i}} \xi=E_{T} \xi
$$

for all $\xi \in L^{2}(\Omega, \mathcal{A}, \mathbb{P})$. The general case now follows by approximation: let $\xi_{t}:=\mathbb{E}\left[\xi \mid \mathcal{A}_{t}\right]$ for all $t \in[0, \infty]$ and note that, given classical stopping times $\left(\tau_{n}\right)_{n \in \mathbb{N}}$ such that $\tau_{n} \rightarrow \tau$ almost surely as $n \rightarrow \infty$,

$$
\mathbb{E}\left[\xi \mid \mathcal{A}_{\tau_{n}}\right]=\xi_{\tau_{n}} \rightarrow \xi_{\tau}=\mathbb{E}\left[\xi \mid \mathcal{A}_{\tau}\right] \quad \text { as } n \rightarrow \infty,
$$

by optional sampling, almost surely and in $L^{2}(\Omega, \mathcal{A}, \mathbb{P})$.

The following theorem has its origins in work of Meyer [28, equation (12) on p.74]; see also [8, Proposition 6], [4, Theorem 6.2] and [19, Theorem 2.5]. This representation shows that $\left(E_{S \wedge s}\right)_{s \in \mathbb{R}_{+}}$is a regular $\Omega$-martingale closed by $E_{S}$; this is a very fruitful observation when combined with the quantum Itô product formula. For the requisite details in regard to quantum stochastic integration, see the appendix, Section A,

Theorem 3.6. Let $S$ be a quantum stopping time. Then

$$
E_{S}=I-\int_{0}^{\infty} I_{\mathrm{k}} \otimes S([0, s]) E_{s} \mathrm{~d} \Lambda_{s}=E_{0}+\int_{0}^{\infty} I_{\mathrm{k}} \otimes S((s, \infty]) E_{s} \mathrm{~d} \Lambda_{s} .
$$

Proof. Note first that

$$
\begin{aligned}
\left\langle\varepsilon(f), \int_{t}^{\infty} I_{\mathrm{k}} \otimes E_{s} \mathrm{~d} \Lambda_{s} \varepsilon(g)\right\rangle & =\int_{t}^{\infty}\langle f(s), g(s)\rangle\left\langle\varepsilon(f), E_{s} \varepsilon(g)\right\rangle \mathrm{d} s \\
& =\int_{t}^{\infty} \frac{\mathrm{d}}{\mathrm{d} t}\left\langle\varepsilon(f), E_{s} \varepsilon(g)\right\rangle \mathrm{d} s \\
& =\left\langle\varepsilon(f),\left(I-E_{t}\right) \varepsilon(g)\right\rangle,
\end{aligned}
$$

so

$$
\begin{aligned}
E_{t} & =I-\int_{t}^{\infty} I_{\mathrm{k}} \otimes E_{s} \mathrm{~d} \Lambda_{s} \\
& =I-\int_{0}^{\infty} I_{\mathrm{k}} \otimes E_{s} \mathrm{~d} \Lambda_{s}+\int_{0}^{t} I_{\mathrm{k}} \otimes E_{s} \mathrm{~d} \Lambda_{s}=E_{0}+\int_{0}^{t} I_{\mathrm{k}} \otimes E_{s} \mathrm{~d} \Lambda_{s} .
\end{aligned}
$$


If $S(\{0\})=I$ then $E_{S}=E_{0}$ and the identities hold as claimed. Now suppose that $S(\{0\})=0$ and let $\pi=\left(\pi_{j}\right)_{j=0}^{\infty}$ be a partition of $\mathbb{R}_{+}$. If $f, g \in L^{2}\left(\mathbb{R}_{+} ; \mathrm{k}\right)$ and $g$ has support in $\left[0, \pi_{n}\right]$ then

$$
\begin{aligned}
\left\langle\varepsilon(f),\left(I-E_{S}^{\pi}\right) \varepsilon(g)\right\rangle & =\sum_{j=1}^{\infty}\left\langle\varepsilon(f), S\left(\left(\pi_{j-1}, \pi_{j}\right]\right)\left(I-E_{\pi_{j}}\right) \varepsilon(g)\right\rangle \\
& =\sum_{j=1}^{n-1} \sum_{k=j}^{n-1}\left\langle\varepsilon(f), S\left(\left(\pi_{j-1}, \pi_{j}\right]\right)\left(E_{\pi_{k+1}}-E_{\pi_{k}}\right) \varepsilon(g)\right\rangle \\
& =\sum_{k=1}^{n-1}\left\langle\varepsilon(f), S\left(\left[0, \pi_{k}\right]\right) \int_{\pi_{k}}^{\pi_{k+1}} I_{\mathrm{k}} \otimes E_{s} \mathrm{~d} \Lambda_{s} \varepsilon(f)\right\rangle \\
& =\left\langle\varepsilon(f),\left(\int_{0}^{\infty} I_{\mathrm{k}} \otimes S([0, s]) E_{s} \mathrm{~d} \Lambda_{s}-R_{\pi}\right) \varepsilon(g)\right\rangle,
\end{aligned}
$$

by Lemma A.6, where

$$
R_{\pi}:=\int_{0}^{\infty} \sum_{k=0}^{\infty} 1_{\left(\pi_{k}, \pi_{k+1}\right]}(s) I_{\mathrm{k}} \otimes S\left(\left(\pi_{k}, s\right]\right) E_{s} \mathrm{~d} \Lambda_{s} .
$$

Finally, if $h \in L^{2}\left(\mathbb{R}_{+} ; \mathbf{k}\right)$ then

$$
\begin{aligned}
\left\|R_{\pi} \varepsilon(h)\right\|^{2} & \leqslant \int_{0}^{\infty}\left\|\sum_{k=0}^{\infty} 1_{\left(\pi_{k}, \pi_{k+1}\right]}(s) S\left(\left(\pi_{k}, s\right]\right) E_{s} \varepsilon(h)\right\|^{2}\|h(s)\|^{2} \mathrm{~d} s \\
& =\int_{0}^{\infty} \sum_{k=0}^{\infty} 1_{\left(\pi_{k}, \pi_{k+1}\right]}(s)\left\|S\left(\left(\pi_{k}, s\right]\right) E_{s} \varepsilon(h)\right\|^{2}\|h(s)\|^{2} \mathrm{~d} s \rightarrow 0
\end{aligned}
$$

as $\pi$ is refined, by the dominated-convergence theorem. To see this, note that if $s \in\left(\pi_{k}, \pi_{k+1}\right]$ then

$$
\begin{aligned}
\left\|S\left(\left(\pi_{k}, s\right]\right) E_{s} \varepsilon(h)\right\|^{2} & =\left\|S\left(\left(\pi_{k}, s\right]\right) \varepsilon(h)\right\|^{2} \exp \left(-\left\|1_{[s, \infty)} h\right\|^{2}\right) \\
& \leqslant\left\|S\left(\left(\pi_{k}, s\right]\right) \varepsilon(h)\right\|^{2} \\
& =\|S([0, s]) \varepsilon(h)\|^{2}-\left\|S\left(\left[0, \pi_{k}\right]\right) \varepsilon(h)\right\|^{2} \rightarrow 0
\end{aligned}
$$

as $s \rightarrow \pi_{k}$, as long as $\pi_{k}$ is not a point of discontinuity of $s \mapsto S([0, s]) \varepsilon(h)$; furthermore,

$$
\sum_{k=0}^{\infty} 1_{\left(\pi_{k}, \pi_{k+1}\right]}(s)\left\|S\left(\left(\pi_{k}, s\right]\right) E_{s} \varepsilon(h)\right\|^{2}\|h(s)\|^{2} \leqslant\left\|E_{s} \varepsilon(h)\right\|^{2}\|h(s)\|^{2}
$$

for all $s \in \mathbb{R}_{+}$. The first identity is now established, and the second may be obtained by writing

$$
I-E_{0}=\int_{0}^{\infty} I_{\mathrm{k}} \otimes E_{s} \mathrm{~d} \Lambda_{s}=\int_{0}^{\infty} I_{\mathrm{k}} \otimes S([0, s]) E_{s} \mathrm{~d} \Lambda_{s}+\int_{0}^{\infty} I_{\mathrm{k}} \otimes S((s, \infty]) E_{s} \mathrm{~d} \Lambda_{s} .
$$


Remark 3.7. Let $S$ be a quantum stopping time. It follows from (3.5) and Theorem A.2 that

$$
\left\|E_{S} x\right\|^{2}=\left\|E_{0} x\right\|^{2}+\int_{0}^{\infty}\left\|\left(I_{\mathrm{k}} \otimes S((s, \infty])\right) D_{s} x\right\|^{2} \mathrm{~d} s \quad \text { for all } x \in \mathcal{F},
$$

where

$$
D: \mathcal{F} \rightarrow L^{2}\left(\mathbb{R}_{+} ; \mathrm{k} \otimes \mathcal{F}\right) ; \quad(D \varepsilon(f))(t)=D_{t} \varepsilon(f):=f(t) \varepsilon\left(1_{[0, t)} f\right)
$$

is the adapted gradient [ 5 , Section 2.2]. When $S$ is deterministic, this identity [5, Proposition 2.3] is a key tool for establishing the existence of quantum stochastic integrals, particularly in the vacuum-adapted setting [14].

Remark 3.8. Let $S$ and $T$ be quantum stopping times. Theorem A.2 and (3.5) imply that

$$
\left\|\left(E_{S}-E_{T}\right) x\right\|^{2}=\int_{0}^{\infty}\left\|\left(I_{\mathrm{k}} \otimes(S([0, s])-T([0, s]))\right) D_{s} x\right\|^{2} \mathrm{~d} s \quad \text { for all } x \in \mathcal{F} .
$$

It follows that the map $S \mapsto E_{S}$ is continuous when the set of time projections is equipped with the strong operator topology and a net of quantum stopping times $\left(S_{\lambda}\right)$ is defined to converge to a spectral measure $S$ (which must then be a quantum stopping time) if and only if $S_{\lambda}([0, t]) \rightarrow S([0, t])$ in the strong operator topology for all but a Lebesgue-null set of points $t$ in $[0, \infty]$; this situation will be denoted by " $S_{\lambda} \Rightarrow S$ ".

Any quantum stopping time $S$ is the limit, in this sense, of a decreasing sequence of discrete quantum stopping times $\left(S_{n}\right)_{n \in \mathbb{N}}$ [30, Proposition 3.3], [12, Proposition 2.3]; a quantum stopping time $S$ is discrete if there exists a finite set $A \subseteq[0, \infty]$, the support of $S$, such that $S(A)=I$ and $S(B) \neq I$ if $B$ is any proper subset of $A$. Note that $S \wedge s \Rightarrow S$ as $s \rightarrow \infty$, so $E_{S \wedge s} \rightarrow E_{S}$ in the strong operator topology.

In [30], Parthasarathy and Sinha employ a weaker notion of discreteness (allowing the support of $S$ to be countably infinite) and a stronger notion of convergence (requiring that $S_{\lambda}([0, t]) \rightarrow$ $S([0, t])$ in the strong operator topology for all $t \in[0, \infty]$ such that $S(\{t\})=0)$.

Example 3.9. [4, pp.507-508], [30, pp.323-324] Recall that the Boson Fock space $\mathcal{F}_{+}(\mathrm{H})$ has a chaos decomposition, so that

$$
\mathcal{F}_{+}(\mathrm{H})=\bigoplus_{n=0}^{\infty} \mathrm{H}^{\otimes_{s} n} \quad \text { and } \quad \varepsilon(f)=\sum_{n=0}^{\infty} \frac{1}{\sqrt{n !}} f^{\otimes n} \quad \text { for all } f \in \mathrm{H},
$$

where $\mathrm{H}$ is any complex Hilbert space and $\otimes_{s}$ denotes the symmetric tensor product.

Fix $n \in \mathbb{Z}_{+}$and, for all $t \in(0, \infty)$, let $P_{n, t} \in B(\mathcal{F})$ be the orthogonal projection onto the subspace

$$
\bigoplus_{j=0}^{n} L^{2}([0, t) ; \mathrm{k})^{\otimes_{s} j} \otimes \mathcal{F}_{[t} \subseteq \mathcal{F}_{t)} \otimes \mathcal{F}_{[t}=\mathcal{F} ;
$$

let $P_{n, 0}=I$, let $P_{n, \infty}=0$ and let $P_{n}$ be the orthogonal projection onto $\bigoplus_{j=0}^{n} L^{2}\left(\mathbb{R}_{+} ; \mathrm{k}\right)^{\otimes_{s} j}$. Note that $P_{n, s} \geqslant P_{n, t}$ for all $s, t \in[0, \infty]$ such that $s \leqslant t$, so setting

$$
S_{n}([0, t]):=P_{n, t}^{\perp}=I-P_{n, t} \quad \text { for all } t \in[0, \infty]
$$


defines a stopping time. Furthermore, if $t \in \mathbb{R}_{+}$and $f \in L^{2}\left(\mathbb{R}_{+} ; \mathrm{k}\right)$ then

$$
S_{n}((t, \infty]) E_{t} \varepsilon(f)=P_{n, t} \varepsilon\left(1_{[0, t)} f\right)=P_{n} E_{t} \varepsilon(f),
$$

so

$$
\begin{aligned}
\left\langle\varepsilon(f), E_{S_{n}} \varepsilon(g)\right\rangle & =1+\int_{0}^{t}\left\langle\varepsilon(f), P_{n} E_{s} \varepsilon(g)\right\rangle\langle f(s), g(s)\rangle \mathrm{d} s \\
& =1+\sum_{k=0}^{n} \frac{1}{k !} \int_{0}^{t}\left(\int_{0}^{s}\langle f(r), g(r)\rangle \mathrm{d} r\right)^{k}\langle f(s), g(s)\rangle \mathrm{d} s \\
& =1+\sum_{k=0}^{n} \frac{1}{(k+1) !}\left(\int_{0}^{t}\langle f(s), g(s)\rangle \mathrm{d} s\right)^{k+1} \\
& =\left\langle\varepsilon(f), P_{n+1} \varepsilon(g)\right\rangle
\end{aligned}
$$

for all $f, g \in L^{2}\left(\mathbb{R}_{+} ; \mathrm{k}\right)$. Thus $E_{S_{n}}=P_{n+1}$.

Finally, note that $P_{n, t} \leqslant P_{n+1, t}$ for all $t \in[0, \infty]$, so $S_{n} \leqslant S_{n+1}$ for all $n \in \mathbb{Z}_{+}$.

Definition 3.10 ([30, Section 5]). If $S$ is a quantum stopping time $S$ then the pre-S space $\left.\mathcal{F}_{S}\right)$ is the range of the time projection $E_{S}$; thus $\mathcal{F}_{S)}:=E_{S}(\mathcal{F})$.

Theorem 3.11 (Cf. [10, Theorem 3.7],[12, Theorem 3.5]). Let $S$ and $T$ be quantum stopping times.

(i) If $S \leqslant T$ then $E_{S} \leqslant E_{T}$ and $\mathcal{F}_{S)} \subseteq \mathcal{F}_{T)}$.

(ii) The time projections $E_{S \wedge T}=E_{S} \wedge E_{T}$ and $E_{S \vee T}=E_{S} \vee E_{T}$.

(iii) If $S([0, t])$ and $T([0, t])$ commute for all $t \in \mathbb{R}_{+}$then so do $E_{S}$ and $E_{T}$.

(iv) If $s \in \mathbb{R}_{+}$then $E_{S} E_{s}=E_{S \wedge s}=E_{s} E_{S}$.

Proof. Some of these claims may be established by working from the definitions, but Theorem 3.6 and the quantum Itô product formula, Theorem A.2, provide a slicker means of obtaining them in the Fock-space context.

(i) As $S([0, s]) T([0, s])=T([0, s])$ for all $s \in[0, \infty]$, it follows that

$$
\begin{aligned}
E_{S} E_{T} & =\left(I-\int_{0}^{\infty} I_{\mathrm{k}} \otimes S([0, s]) E_{s} \mathrm{~d} \Lambda_{s}\right)\left(I-\int_{0}^{\infty} I_{\mathrm{k}} \otimes T([0, s]) E_{s} \mathrm{~d} \Lambda_{s}\right) \\
& =I-\int_{0}^{\infty} I_{\mathrm{k}} \otimes\left(S([0, s]) E_{s}+T([0, s]) E_{s}-S([0, s]) T([0, s]) E_{s}\right) \mathrm{d} \Lambda_{s} \\
& =I-\int_{0}^{\infty} I_{\mathrm{k}} \otimes S([0, s]) E_{s} \mathrm{~d} \Lambda_{s} \\
& =E_{S} .
\end{aligned}
$$


(ii) By the quantum Itô product formula and von Neumann's method of alternating projections,

$$
\begin{aligned}
\left(E_{S}^{\perp} E_{T}^{\perp}\right)^{n} & =\int_{0}^{\infty} I_{\mathrm{k}} \otimes\left((S([0, s]) T([0, s]))^{n} E_{s} \mathrm{~d} \Lambda_{s}\right. \\
& \rightarrow \int_{0}^{\infty} I_{\mathrm{k}} \otimes(S \wedge T)([0, s]) E_{s} \mathrm{~d} \Lambda_{s}=E_{S \vee T}^{\perp}
\end{aligned}
$$

as $n \rightarrow \infty$, so $E_{S \vee T}=\left(E_{S}^{\perp} \wedge E_{T}^{\perp}\right)^{\perp}=E_{S} \vee E_{T}$.

For the second identity, note first that $E_{S}-E_{0}$ and $E_{T}-E_{0}$ are orthogonal projections, by (i), and, as $n \rightarrow \infty$,

$$
\begin{aligned}
\left(\left(E_{S}-E_{0}\right)\left(E_{T}-E_{0}\right)\right)^{n} & =\int_{0}^{\infty} I_{\mathrm{k}} \otimes(S((s, \infty]) T((s, \infty]))^{n} E_{s} \mathrm{~d} \Lambda_{s} \\
& \rightarrow \int_{0}^{\infty} I_{\mathrm{k}} \otimes(S \wedge T)((s, \infty]) E_{s} \mathrm{~d} \Lambda_{s} \\
& =E_{S \wedge T}-E_{0} .
\end{aligned}
$$

However, as $E_{S} E_{0}=E_{0} E_{S}=E_{0}=E_{0} E_{T}=E_{T} E_{0}$, we also have that

$$
\left(\left(E_{S}-E_{0}\right)\left(E_{T}-E_{0}\right)\right)^{n}=\left(E_{S} E_{T}-E_{0}\right)^{n}=\left(E_{S} E_{T}\right)^{n}-E_{0} \rightarrow E_{S} \wedge E_{T}-E_{0},
$$

which gives the result.

(iii) As $S([0, s]) T([0, s])=T([0, s]) S([0, s])$ for all $s \in \mathbb{R}_{+}$, it follows that

$$
E_{S} E_{T}=I-\int_{0}^{\infty} I_{\mathrm{k}} \otimes(S([0, s])+T([0, s])-S([0, s]) T([0, s])) E_{s} \mathrm{~d} \Lambda_{s}=E_{T} E_{S} .
$$

(iv) By (ii) and (iii), as $S$ and $s$ commute as required, so $E_{S} \wedge E_{s}=E_{S} E_{s}$.

Proposition 3.12. For all $s \in[0, \infty]$, it holds that

$$
E_{S \wedge s}=S([0, s]) E_{S}+S((s, \infty]) E_{s} .
$$

Proof. Without loss of generality, let $s \in \mathbb{R}_{+}$and let $\pi$ be a partition of $\mathbb{R}_{+}$with $s=\pi_{n}$ for some $n \in \mathbb{Z}_{+}$. Then

$$
\begin{aligned}
E_{S}^{\pi} E_{s} & =S(\{0\}) E_{0}+\sum_{j=1}^{n} S\left(\left(\pi_{j-1}, \pi_{j}\right]\right) E_{\pi_{j}}+\sum_{j=n+1}^{\infty} S\left(\left(\pi_{j-1}, \pi_{j}\right]\right) E_{s} \\
& +S(\{\infty\}) E_{s} \\
& =S([0, s]) E_{S}^{\pi}+S((s, \infty]) E_{s} .
\end{aligned}
$$

The claim now follows by refining $\pi$, since $E_{S} E_{s}=E_{S \wedge s}$ by Theorem 3.11(iii). 


\section{The stopping algebras}

Definition 4.1. Given $Z \in B(\mathcal{F})$ and a quantum stopping time $S$, let

$$
Z_{\breve{S}}:=E_{S} Z E_{S} \in B(\mathcal{F})
$$

Note that $E_{S} Z_{\breve{S}} E_{S}=Z_{\breve{S}} E_{S}$, and so $Z_{\breve{S}}$ maps $\mathcal{F}_{S)}$ to itself. Remark 3.8 implies that the mapping $(Z, S) \mapsto Z_{\breve{S}}$ is jointly continuous on the product of any bounded subset of $B(\mathcal{F})$ with the collection of all quantum stopping times, when $B(\mathcal{F})$ is equipped with the strong operator topology and a net $\left(S_{\lambda}\right)$ of quantum stopping times converges to the quantum stopping time $S$ if and only if $S_{\lambda} \Rightarrow S$, as defined in Remark 3.8.

Proposition 4.2. The map $Z \mapsto Z_{\breve{S}}$ is a conditional expectation from $B(\mathcal{F})$ onto the normclosed $*$-subalgebra $B_{\breve{S}}:=\left\{E_{S} Z E_{S}: Z \in B(\mathcal{F})\right\}$ that preserves the vacuum state

$$
\mathbb{E}_{\Omega}: B(\mathcal{F}) \rightarrow \mathbb{C} ; Z \mapsto\langle\varepsilon(0), Z \varepsilon(0)\rangle .
$$

Proof. This is a straightforward exercise.

Remark 4.3. The collection of stopped algebras

$$
\left\{B_{\breve{S}}: S \text { is a quantum stopping time }\right\}
$$

has the following properties.

(i) If $S$ is a quantum stopping time and $Z \in B_{\breve{S}}$ then $E_{S} Z E_{S}=Z E_{S}$, so $Z$ preserves the pre- $S$ space $\mathcal{F}_{S \text { ) }}$.

(ii) If the quantum stopping times $S$ and $T$ satisfy $S \leqslant T$ then $B_{\breve{S}} \subseteq B_{\breve{T}}$, by Theorem 3.11 (i).

(iii) For deterministic stopping times,

$$
B_{\check{0}}=\operatorname{im} P_{[0}^{\Omega}, \quad B_{\breve{t}}=B\left(\mathcal{F}_{t)}\right) \otimes P_{[t}^{\Omega} \quad \text { and } \quad B_{\check{\infty}}=B(\mathcal{F})
$$

for all $t \in(0, \infty)$, where $P_{[s}^{\Omega} \in B\left(\mathcal{F}_{[s}\right)$ is the orthogonal projection onto the vacuum subspace $\mathbb{C} \varepsilon\left(\left.0\right|_{[s, \infty)}\right)$ for all $s \in \mathbb{R}_{+}$.

As noted by Coquio, this is impossible if we work instead in the identity-adapted setting with the natural analogue of (iii): see [19, Proposition 2.2].

\section{$5 \quad$ Processes and martingales}

Definition 5.1. A process is a family $X=\left(X_{t}\right)_{t \in \mathbb{R}_{+}} \subseteq B(\mathcal{F})$. (We have no need to impose any measurability or adaptedness conditions at this point.) Two processes $X$ and $Y$ are equal if and only if $X_{t}=Y_{t}$ for all $t \in \mathbb{R}_{+}$. The set of processes is a complex associative *-algebra, where addition, multiplication and the adjoint are defined pointwise. 
Given $Z \in B(\mathcal{F})$, let $\breve{\pi}(Z)$ and $\widehat{\pi}(Z)$ be the processes with initial values

$$
\check{\pi}(Z)_{0}=\mathbb{E}_{\Omega}[Z] E_{0} \quad \text { and } \quad \widehat{\pi}(Z)_{0}=\mathbb{E}_{\Omega}[Z] I,
$$

and such that

$$
\check{\pi}(Z)_{t}=E_{t} Z E_{t} \quad \text { and } \quad \widehat{\pi}(Z)_{t}=\left.E_{t} Z\right|_{\mathcal{F}_{t)}} \otimes I_{[t} \quad \text { for all } t \in(0, \infty) .
$$

Note that $t \mapsto \breve{\pi}(Z)_{t}$ and $t \mapsto \widehat{\pi}(Z)_{t}$ are uniformly bounded and continuous on $[0, \infty]$ in the strong operator topology, where $\breve{\pi}(Z)_{\infty}=\widehat{\pi}(Z)_{\infty}:=Z$, for any $Z \in B(\mathcal{F})$. Note also that the maps $Z \mapsto \breve{\pi}(Z)$ and $Z \mapsto \widehat{\pi}(Z)$ are $*$-algebra homomorphisms. We extend these definitions from operators to processes by setting

$$
\check{\pi}(X)_{t}:=\check{\pi}\left(X_{t}\right)_{t} \quad \text { and } \quad \widehat{\pi}(X)_{t}=\widehat{\pi}\left(X_{t}\right)_{t} \quad \text { for all } t \in[0, \infty],
$$

where $X$ is an arbitrary process; if not otherwise defined, we let $X_{\infty}:=0$. For convenience, we will also consider processes indexed by $[0, \infty]$.

A process $X$ is adapted if $X_{t} E_{t}=E_{t} X_{t}$ for all $t \in \mathbb{R}_{+}$.

A process $X$ is vacuum adapted if $X=\breve{\pi}(X)$; this is equivalent to the requirement that $X_{t} E_{t}=$ $X_{t}=E_{t} X_{t}$ for all $t \in \mathbb{R}_{+}$. Note that $\breve{\pi}(Z)$ is a vacuum-adapted process for any $Z \in B(\mathcal{F})$.

A process $X$ is identity adapted if $X=\widehat{\pi}(X)$. Note that $\widehat{\pi}(Z)$ is an identity-adapted process for any $Z \in B(\mathcal{F})$.

Vacuum-adapted and identity-adapted processes are adapted, and the sets of adapted processes, vacuum-adapted processes and identity-adapted processes are $*$-subalgebras of the $*$-algebra of processes.

The process $M$ is a martingale if it is adapted and $E_{s} M_{t} E_{s}=M_{s} E_{s}$ for all $s, t \in \mathbb{R}_{+}$with $s \leqslant t$.

The martingale $M$ is closed if $\operatorname{st}_{t \rightarrow \infty} M_{t}=M_{\infty}$ for some $M_{\infty} \in B(\mathcal{F})$, where "st.lim" denotes the limit in the strong operator topology.

The sets of martingales and closed martingales are subspaces of the algebra of adapted processes.

Proposition 5.2. A process $X$ is a vacuum-adapted martingale closed by $X_{\infty}$ if and only if $X=\check{\pi}\left(X_{\infty}\right)$.

Proof. Suppose $X=\breve{\pi}\left(X_{\infty}\right)$. Then $X$ is vacuum adapted, and if $s, t \in \mathbb{R}_{+}$are such that $s \leqslant t$ then

$$
E_{s} X_{t} E_{s}=E_{s} E_{t} X_{\infty} E_{t} E_{s}=E_{s} X_{\infty} E_{s}=X_{s}=X_{s} E_{s},
$$

so $X$ is a martingale. Furthermore, $X_{t}=\breve{\pi}\left(X_{\infty}\right)_{t} \rightarrow \breve{\pi}\left(X_{\infty}\right)_{\infty}=X_{\infty}$ in the strong operator topology as $t \rightarrow \infty$, so $X$ is closed by $X_{\infty}$.

Conversely, if $X$ is a vacuum-adapted martingale and $X_{\infty}=\operatorname{st}_{t \rightarrow \infty} X_{t}$ then, for all $s \in \mathbb{R}_{+}$,

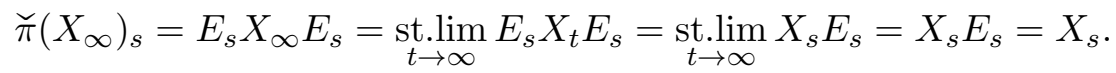


Proposition 5.3. A process $X$ is an identity-adapted martingale closed by $X_{\infty}$ if and only if $X=\widehat{\pi}\left(X_{\infty}\right)$.

Proof. Suppose $X=\widehat{\pi}\left(X_{\infty}\right)$. Then $X$ is identity adapted, and if $s, t \in \mathbb{R}_{+}$are such that $s \leqslant t$ then

$$
E_{s} X_{t} E_{s}=E_{s} \widehat{\pi}\left(X_{\infty}\right)_{t} E_{s}=E_{s} X_{\infty} E_{s}=\widehat{\pi}\left(X_{\infty}\right)_{s} E_{s}
$$

so $X$ is a martingale. Furthermore, $X_{t}=\widehat{\pi}\left(X_{\infty}\right)_{t} \rightarrow \widehat{\pi}\left(X_{\infty}\right)_{\infty}=X_{\infty}$ in the strong operator topology as $t \rightarrow \infty$, so $X$ is closed by $X_{\infty}$.

Conversely, if $X$ is an identity-adapted martingale and $X_{\infty}=\underset{t \rightarrow \infty}{\operatorname{st} . \lim } X_{t}$ then, for all $s \in \mathbb{R}_{+}$,

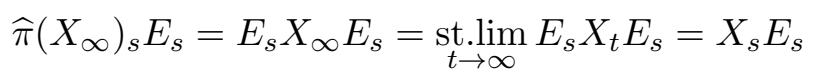

and

$$
\widehat{\pi}\left(X_{\infty}\right)_{s}=\widehat{\pi}\left(\widehat{\pi}\left(X_{\infty}\right)_{s} E_{s}\right)_{s}=\widehat{\pi}\left(X_{s} E_{s}\right)_{s}=\widehat{\pi}\left(X_{s}\right)_{s}=X_{s} .
$$

Proposition 5.4. If $Z \in B(\mathcal{F})$ and $S$ is a quantum stopping time then the process $Z^{\breve{S}}:=$ $\left(Z_{\widetilde{S \wedge t}}\right)_{t \in \mathbb{R}_{+}}$is a vacuum-adapted martingale closed by $Z_{\breve{S}}$.

Proof. If $t \in \mathbb{R}_{+}$then Theorem 3.11(ii) implies that

$$
E_{t} Z_{\breve{S}} E_{t}=E_{t} E_{S} Z E_{S} E_{t}=E_{S \wedge t} Z E_{S \wedge t}=Z_{t}^{\check{S}},
$$

so the result follows from Proposition 5.2 .

Remark 5.5. The process $Z^{\breve{S}}$ remains a vacuum-adapted martingale if $\left(E_{t}\right)_{t \in \mathbb{R}_{+}}$is replaced by $\left(E_{S \wedge t}\right)_{t \in \mathbb{R}_{+}}$in Definition [5.1, as the analogue of Proposition 5.2 holds.

\section{$6 \quad$ Stopping processes at discrete times}

Definition 6.1. A quantum stopping time $T$ is said to be discrete if there exists a finite set of times $\left\{t_{1}<\ldots<t_{n}\right\} \subseteq[0, \infty]$, called the support of $T$, such that $T\left(\left\{t_{i}\right\}\right) \neq 0$ for $i=1, \ldots, n$ and $T\left(\left\{t_{1}, \ldots, t_{n}\right\}\right)=I$. Note that

$$
T([0, t])=\left\{\begin{array}{cl}
0 & \text { if } t<t_{1}, \\
T\left(\left\{t_{1}, \ldots, t_{m}\right\}\right) & \text { if } t \in\left[t_{m}, t_{m+1}\right) \quad(m=1, \ldots, n-1), \\
I & \text { if } t \geqslant t_{n}
\end{array}\right.
$$

for all $t \in[0, \infty]$. In particular, $t_{1} \leqslant T \leqslant t_{n}$. 
Definition 6.2. If $X$ is a process and $T$ is a discrete quantum stopping time with support $\left\{t_{1}, \ldots, t_{n}\right\}$ then Coquio [19, Definition 3.2(2)] stops $X$ at $T$ by setting

$$
\begin{aligned}
X_{\widehat{T}} & :=\sum_{i, j=1}^{n} \widehat{\pi}\left(E_{t_{i}}\right)_{t_{i} \vee t_{j}} T_{i} \widehat{\pi}(X)_{t_{i} \vee t_{j}} T_{j} \widehat{\pi}\left(E_{t_{j}}\right)_{t_{i} \vee t_{j}} \\
& =\sum_{i, j=1}^{n} \widehat{\pi}\left(E_{t_{i}} T_{i} X_{t_{i} \vee t_{j}} T_{j} E_{t_{j}}\right)_{t_{i} \vee t_{j}},
\end{aligned}
$$

where $T_{i}:=T\left(\left\{t_{i}\right\}\right)$ for $i=1, \ldots n$. Recall that $X_{\infty}:=0$ if it is not otherwise defined.

Note that if $t \in[0, \infty]$ then $X_{\widehat{t}}=\widehat{\pi}(X)_{t}$. Furthermore, $E_{\widehat{T}}=I$ and, considering $I$ as a constant process, $I_{\widehat{T}}=I$.

Remark 6.3. Coquio only considers identity-adapted processes, for which her definition agrees with Definition 6.2 above. She prefers the notation $M_{T}(Z)$ when stopping an operator; as we do not require processes to be adapted, so may identify operators with constant processes, our choice of notation seems more convenient.

Definition 6.4. Let $X$ be a process and let $T$ be a discrete quantum stopping time with support $\left\{t_{1}<\ldots<t_{n}\right\}$. The result of applying vacuum-adapted stopping to $X$ at $T$ is

$$
X_{\breve{T}}:=\sum_{i, j=1}^{n} E_{t_{i}} T_{i} X_{t_{i} \vee t_{j}} T_{j} E_{t_{j}}=\sum_{i, j=1}^{n} T_{i} E_{t_{i}} X_{t_{i} \vee t_{j}} E_{t_{j}} T_{j},
$$

where $T_{i}:=T\left(\left\{t_{i}\right\}\right)$ for $i=1, \ldots, n$; again, let $X_{\infty}:=0$ if necessary. This is analogous to Coquio's definition for the identity-adapted case.

Note that if $t \in[0, \infty]$ then $X_{\breve{t}}=\breve{\pi}(X)_{t}$. Furthermore, if the process $X$ has the constant value $Z$ then $X_{\breve{T}}=E_{T} Z E_{T}=Z_{\breve{T}}$ in the sense of Definition 4.1, in particular, $I_{\breve{T}}=E_{T}$. It also follows from the definition that $E_{\breve{T}}=E_{T}$.

Proposition 6.5. If $X$ is a process and $T$ is a discrete quantum stopping time then

$$
\left(X_{\breve{T}}\right)_{\breve{T}}=X_{\breve{T}}, \quad\left(X_{\widehat{T}}\right)_{\breve{T}}=X_{\breve{T}}, \quad\left(X_{\breve{T}}\right)_{\widehat{T}}=X_{\widehat{T}} \quad \text { and } \quad\left(X_{\widehat{T}}\right)_{\widehat{T}}=X_{\widehat{T}},
$$

where $X_{\widehat{T}}$ and $X_{\breve{T}}$ are stopped by regarding them as constant processes.

Proof. This follows because $E_{r} \widehat{\pi}\left(E_{r}\right)_{s}=E_{r}=\widehat{\pi}\left(E_{r}\right)_{s} E_{r}$ for all $r, s \in[0, \infty]$ such that $r \leqslant s$, and $E_{r} \widehat{\pi}\left(X_{r \vee s}\right)_{r \vee s} E_{s}=E_{r} X_{r \vee s} E_{s}$ for all $r, s \in[0, \infty]$.

Proposition 6.6. If $T$ is a discrete quantum stopping time and $X$ is a process then

$$
T([0, t]) X_{\check{T}} T([0, t])=T([0, t]) X_{\widetilde{T \wedge t}} T([0, t]),
$$

whereas

$$
X_{\widehat{T}} E_{T}=E_{T} X_{\widehat{T}}=X_{\breve{T}} \quad \text { and } \quad T([0, t]) X_{\widehat{T}} T([0, t])=T([0, t]) X_{\widehat{T \wedge t}} T([0, t]),
$$

for all $t \in[0, \infty]$. 
Proof. Without loss of generality, suppose that $t \in\left[t_{k}, t_{k+1}\right)$, where $t_{0}:=0$ and $t_{n+1}:=\infty$. Since $T([0, t])(T \wedge t)(\{t\})=T(\{t\})$ and the support of $T \wedge t$ has maximum element $t$, it follows that

$$
T([0, t]) X_{\breve{T}} T([0, t])=\sum_{i, j=1}^{k} E_{t_{i}} T_{i} X_{t_{i} \vee t_{j}} T_{j} E_{t_{j}}=T([0, t]) X_{\widetilde{T \wedge t}} T([0, t]) .
$$

The other identities are contained in [19, Properties 3.3(1-2)].

Proposition 6.7. If $T$ is a discrete quantum stopping time and $X$ is a process then

$$
\check{\pi}\left(X_{\widetilde{T \wedge t}}\right)_{t}=X_{\widetilde{T \wedge t}} \quad \text { and } \quad \widehat{\pi}\left(X_{\widehat{T \wedge t}}\right)_{t}=X_{\widehat{T \wedge t}} \quad \text { for all } t \in[0, \infty] \text {, }
$$

so the processes $X^{\check{T}}:=\left(X_{\widehat{T \wedge t}}\right)_{t \in \mathbb{R}_{+}}$and $X^{\widehat{T}}:=\left(X_{\widehat{T \wedge t}}\right)_{t \in \mathbb{R}_{+}}$are vacuum adapted and identity adapted, respectively.

Proof. Since $T \wedge t$ is discrete and $T \wedge t \leqslant t$, by Theorem 2.11, it follows from the first part of Proposition 6.6 and Theorem 3.11(i) that

$$
E_{t} X_{\widetilde{T \wedge t}} E_{t}=E_{t} E_{T \wedge t} X_{\widetilde{T \wedge t}} E_{T \wedge t} E_{t}=E_{T \wedge t} X_{\widetilde{T \wedge t}} E_{T \wedge t}=X_{\overparen{T \wedge t}} .
$$

The second claim is contained in [19, Properties 3.3(2)].

Remark 6.8. The definitions of $X^{\breve{T}}$ and $Z^{\check{S}}$ in Propositions 6.7 and 5.4 are consistent: they agree when when $S=T$ is discrete and $Z$ is regarded as a constant process $X$, by the penultimate remark in Definition 6.4

Lemma 6.9. If $M$ is a martingale and $T$ is a discrete quantum stopping time with support $\left\{t_{1}<\cdots<t_{n}\right\}$ then $M_{\breve{T}}=E_{T} M_{t} E_{T}$ for all $t \in\left[t_{n}, \infty\right)$.

Proof. With the notation of Definition 6.4, if $t \in\left[t_{n}, \infty\right)$ then

$$
M_{\breve{T}}=\sum_{i, j=1}^{n} T_{i} E_{t_{i}} E_{t_{i} \vee t_{j}} M_{t} E_{t_{i} \vee t_{j}} E_{t_{j}} T_{j}=\sum_{i, j=1}^{n} T_{i} E_{t_{i}} M_{t} E_{t_{j}} T_{j}=E_{T} M_{t} E_{T} .
$$

Theorem 6.10. Let $X$ be an adapted process. Then $X$ is a martingale if and only if $\mathbb{E}_{\Omega}\left[X_{\breve{T}}\right]=$ $\mathbb{E}_{\Omega}\left[X_{0}\right]$ for every discrete quantum stopping time $T$, where $\mathbb{E}_{\Omega}$ is the vacuum state (4.1).

Proof. We follow the proof of [19, Proposition 3.10]. If $X$ is a martingale and the discrete quantum stopping time $T$ has support $\left\{t_{1}<\cdots<t_{n}\right\}$ then Lemma 6.9 implies that

$$
\mathbb{E}_{\Omega}\left[X_{\breve{T}}\right]=\left\langle\varepsilon(0), E_{0} E_{T} X_{t_{n}} E_{T} E_{0} \varepsilon(0)\right\rangle=\left\langle\varepsilon(0), E_{0} X_{t_{n}} E_{0} \varepsilon(0)\right\rangle=\mathbb{E}_{\Omega}\left[X_{0}\right] ;
$$

note that $T \wedge 0=0$ and $E_{0} E_{T}=E_{0}=E_{T} E_{0}$, by Theorem 3.11 (ii). 
Conversely, let $T$ have support $\{s<t\} \subseteq \mathbb{R}_{+}$, let $T(\{s\})=P$ and note that

$$
\begin{aligned}
\mathbb{E}_{\Omega}\left[X_{\check{T}}\right] & =\mathbb{E}_{\Omega}\left[P X_{s} P\right]+\mathbb{E}_{\Omega}\left[P X_{t} P^{\perp}\right]+\mathbb{E}_{\Omega}\left[P^{\perp} X_{t} P\right]+\mathbb{E}_{\Omega}\left[P^{\perp} X_{t} P^{\perp}\right] \\
& =\mathbb{E}_{\Omega}\left[P\left(X_{s}-X_{t}\right) P+X_{t}\right],
\end{aligned}
$$

so if $\mathbb{E}_{\Omega}\left[X_{\breve{T}}\right]=\mathbb{E}_{\Omega}\left[X_{0}\right]=\mathbb{E}_{\Omega}\left[X_{\breve{t}}\right]=\mathbb{E}_{\Omega}\left[X_{t}\right]$ then

$$
\left\langle P \varepsilon(0),\left(X_{t}-X_{s}\right) P \varepsilon(0)\right\rangle=0
$$

for any orthogonal projection $P \in B\left(\mathcal{F}_{s)}\right) \otimes I_{[s}$, so for any $P \in B\left(\mathcal{F}_{s)}\right) \otimes I_{[s}$. It follows that $E_{s}\left(X_{t}-X_{s}\right) E_{s}=0$, as required.

Remark 6.11. Since $\mathbb{E}_{\Omega}\left[X_{\breve{T}}\right]=\mathbb{E}_{\Omega}\left[X_{\widehat{T}}\right]$ for any process $X$ and any discrete quantum stopping time $T$, the identity-adapted version of Theorem 6.10 holds: Coquio's work contains a similar result [19, Proposition 3.10].

The following result expresses the relationship between the vacuum and identity-adapted martingales closed by the same operator; cf. [19, Lemma 3.6]. It is provides the key to stopping processes for more general times.

Lemma 6.12. If $X$ is a process and $t \in[0, \infty]$ then

$$
\widehat{\pi}(X)_{t}=\check{\pi}(X)_{t}+\int_{t}^{\infty} I_{\mathrm{k}} \otimes \widehat{\pi}\left(\breve{\pi}(X)_{t}\right)_{s} \mathrm{~d} \Lambda_{s}=\check{\pi}(X)_{t}+\int_{t}^{\infty} I_{\mathrm{k}} \otimes \check{\pi}\left(\widehat{\pi}(X)_{t}\right)_{s} \mathrm{~d} \Lambda_{s} .
$$

Proof. Applying Theorem A.8 to the first identity in (3.6), it follows that

$$
I-E_{t}=\widehat{\pi}\left(I-E_{t}\right)_{\infty}=\int_{t}^{\infty} I_{\mathrm{k}} \otimes\left(I-\widehat{\pi}\left(I-E_{t}\right)_{s}\right) \mathrm{d} \Lambda_{s}=\int_{t}^{\infty} I_{\mathrm{k}} \otimes \widehat{\pi}\left(E_{t}\right)_{s} \mathrm{~d} \Lambda_{s} .
$$

Hence, by Lemma A.6,

$$
\widehat{\pi}(X)_{t}=\widehat{\pi}(X)_{t}\left(E_{t}+\int_{t}^{\infty} I_{\mathrm{k}} \otimes \widehat{\pi}\left(E_{t}\right)_{s} \mathrm{~d} \Lambda_{s}\right)=\breve{\pi}(X)_{t}+\int_{t}^{\infty} I_{\mathrm{k}} \otimes \widehat{\pi}\left(\breve{\pi}(X)_{t}\right)_{s} \mathrm{~d} \Lambda_{s} .
$$

Similar working, but using the first identity in (3.6) directly, gives the second claim.

Remark 6.13. It does not follow from Lemma 6.12 that $\widehat{\pi}\left(\breve{\pi}(X)_{t}\right)_{s}=\breve{\pi}\left(\widehat{\pi}(X)_{t}\right)_{s}$ for almost all $s \geqslant t$. This is because the integrands are adapted in difference senses, so their difference is neither identity adapted nor vacuum adapted, in general; thus Remark A.5 and Lemma A.7 cannot be applied. The same situation occurs repeatedly below; for example, see Theorem 6.14.

The following theorem extends Lemma 6.12 from deterministic to discrete quantum stopping times. If it is known how to stop a class of processes for one form of adaptedness, this result provides stopping of that class for the other form. The identity-adapted version for closed martingales was obtained by Coquio [19, Proof of Theorem 3.5]. 
Theorem 6.14. If $X$ is a process and $T$ is a discrete quantum stopping time then

$$
\begin{aligned}
X_{\widehat{T}} & =X_{\breve{T}}+\int_{0}^{\infty} I_{\mathrm{k}} \otimes T([0, s]) \widehat{\pi}\left(X_{\breve{T}}\right)_{s} T([0, s]) \mathrm{d} \Lambda_{s} \\
& =X_{\breve{T}}+\int_{0}^{\infty} I_{\mathrm{k}} \otimes T([0, s]) \check{\pi}\left(X_{\widehat{T}}\right)_{s} T([0, s]) \mathrm{d} \Lambda_{s} .
\end{aligned}
$$

Proof. If $T$ has support $\left\{t_{1}<\cdots<t_{n}\right\}$ and $T_{i}=T\left(\left\{t_{i}\right\}\right)$ for $i=1, \ldots, n$ then, by Lemmas 6.12 and A.6.

$$
\begin{aligned}
X_{\widehat{T}}= & \sum_{i, j=1}^{n} T_{i} \widehat{\pi}\left(E_{t_{i}}\right)_{t_{i} \vee t_{j}} \widehat{\pi}(X)_{t_{i} \vee t_{j}} \widehat{\pi}\left(E_{t_{j}}\right)_{t_{i} \vee t_{j}} T_{j} \\
= & \sum_{i, j=1}^{n}\left(T_{i} \widehat{\pi}\left(E_{t_{i}}\right)_{t_{i} \vee t_{j}} \check{\pi}(X)_{t_{i} \vee t_{j}} \widehat{\pi}\left(E_{t_{j}}\right)_{t_{i} \vee t_{j}} T_{j}\right. \\
& \left.\quad+\int_{t_{i} \vee t_{j}}^{\infty} I_{\mathrm{k}} \otimes T_{i} \widehat{\pi}\left(E_{t_{i}}\right)_{t_{i} \vee t_{j}} \widehat{\pi}\left(\check{\pi}(X)_{t_{i} \vee t_{j}}\right)_{s} \widehat{\pi}\left(E_{t_{j}}\right)_{t_{i} \vee t_{j}} T_{j} \mathrm{~d} \Lambda_{s}\right) \\
= & \sum_{i, j=1}^{n}\left(T_{i} E_{t_{i}} X_{t_{i} \vee t_{j}} E_{t_{j}} T_{j}\right. \\
& \left.\quad+\int_{0}^{\infty} I_{\mathrm{k}} \otimes 1_{\left[t_{i}, \infty\right)}(s) 1_{\left[t_{j}, \infty\right)}(s) T_{i} \widehat{\pi}\left(E_{t_{i}}\right)_{s} \widehat{\pi}\left(X_{t_{i} \vee t_{j}}\right)_{s} \widehat{\pi}\left(E_{t_{j}}\right)_{s} T_{j} \mathrm{~d} \Lambda_{s}\right) \\
= & X_{\breve{T}}+\int_{0}^{\infty} I_{\mathrm{k}} \otimes T([0, s]) \widehat{\pi}\left(X_{\breve{T}}\right)_{s} T([0, s]) \mathrm{d} \Lambda_{s} ;
\end{aligned}
$$

the penultimate equality holds because $\widehat{\pi}\left(E_{r}\right)_{s} \widehat{\pi}\left(E_{s}\right)_{t}=\widehat{\pi}\left(E_{r}\right)_{t}$ if $r \leqslant s \leqslant t$, and the final equality holds because

$$
\begin{aligned}
T([0, s]) \widehat{\pi} & \left(X_{\breve{T}}\right)_{s} T([0, s]) \\
& =\sum_{i, j, k, l=1}^{n} 1_{\left[t_{i}, \infty\right)}(s) 1_{\left[t_{j}, \infty\right)}(s) T_{i} \widehat{\pi}\left(T_{k} E_{t_{k}} X_{t_{k} \vee t_{l}} E_{t_{l}} T_{l}\right)_{s} T_{j} \\
& =\sum_{i, j, k, l=1}^{n} 1_{\left[t_{i}, \infty\right)}(s) 1_{\left[t_{j}, \infty\right)}(s) \widehat{\pi}\left(T_{i} T_{k}\right)_{s} \widehat{\pi}\left(E_{t_{k}} X_{t_{k} \vee t_{l}} E_{t_{l}}\right)_{s} \widehat{\pi}\left(T_{l} T_{j}\right)_{s} \\
& =\sum_{i, j=1}^{n} 1_{\left[t_{i}, \infty\right)}(s) 1_{\left[t_{j}, \infty\right)}(s) T_{i} \widehat{\pi}\left(E_{t_{i}}\right)_{s} \widehat{\pi}\left(X_{t_{i} \vee t_{j}}\right)_{s} \widehat{\pi}\left(E_{t_{j}}\right)_{s} T_{j} .
\end{aligned}
$$


Similarly, using the second identity in Lemma 6.12,

$$
\begin{aligned}
X_{\widehat{T}}-X_{\breve{T}} & =\sum_{i, j=1}^{n} \int_{t_{i} \vee t_{j}}^{\infty} I_{\mathrm{k}} \otimes T_{i} \widehat{\pi}\left(E_{t_{i}}\right)_{t_{i} \vee t_{j}} \breve{\pi}\left(\widehat{\pi}(X)_{t_{i} \vee t_{j}}\right)_{s} \widehat{\pi}\left(E_{t_{j}}\right)_{t_{i} \vee t_{j}} T_{j} \mathrm{~d} \Lambda_{s} \\
& =\sum_{i, j=1}^{n} \int_{0}^{\infty} I_{\mathrm{k}} \otimes 1_{\left[t_{i} \vee t_{j}, \infty\right)}(s) E_{s} T_{i} \widehat{\pi}\left(E_{t_{i}} X_{t_{i} \vee t_{j}} E_{t_{j}}\right)_{t_{i} \vee t_{j}} T_{j} E_{s} \mathrm{~d} \Lambda_{s} \\
& =\int_{0}^{\infty} I_{\mathrm{k}} \otimes T([0, s]) \check{\pi}\left(X_{\widehat{T}}\right)_{s} T([0, s]) \mathrm{d} \Lambda_{s},
\end{aligned}
$$

since if $s \geqslant t_{i} \vee t_{j}$ then

$$
\begin{aligned}
T_{i} \breve{\pi}\left(X_{\widehat{T}}\right)_{s} T_{j} & =\sum_{k, l=1}^{n} E_{s} T_{i} T_{k} \widehat{\pi}\left(E_{t_{k}} X_{t_{k} \vee t_{l}} E_{t_{l}}\right)_{t_{k} \vee t_{l}} T_{l} T_{j} E_{s} \\
& =E_{s} T_{i} \widehat{\pi}\left(E_{t_{i}} X_{t_{i} \vee t_{j}} E_{t_{j}}\right) T_{j} E_{s} .
\end{aligned}
$$

Remark 6.15. The integrals on the right-hand sides of (6.1) and (6.2) may be viewed as artifacts produced by working with identity-adapted processes rather than vacuum-adapted ones.

\section{Stopping martingales at general times}

Definition 7.1. If $M=\left(M_{t}\right)_{t \in \mathbb{R}_{+}}$is a martingale closed by $M_{\infty}$ and $T$ is a discrete quantum stopping time with support $\left\{t_{1}<\cdots<t_{n}\right\}$ then Lemma 6.9 implies that

$$
M_{\breve{T}}=E_{T} M_{t_{n}} E_{T}=E_{T} M_{t_{n}} E_{t_{n}} E_{T}=E_{T} E_{t_{n}} M_{\infty} E_{t_{n}} E_{T}=E_{T} M_{\infty} E_{T}
$$

Hence the result of applying vacuum-adapted stopping to a martingale $M$ closed by $M_{\infty}$ at a quantum stopping time $S$ is defined to be

$$
M_{\breve{S}}:=E_{S} M_{\infty} E_{S} .
$$

Note that $E=\left(E_{t}\right)_{t \in \mathbb{R}_{+}}$is a vacuum-adapted martingale closed by $E_{\infty}=I$ and $I_{\breve{S}}=E_{S}$ for any quantum stopping time $S$. Furthermore, the map $S \mapsto M_{\breve{S}}$ is continuous if the collection of quantum stopping times is equipped with the topology described in Remark 3.8 and $B(\mathcal{F})$ has the strong operator topology.

Theorem 7.2. Let $M$ be a closed martingale and let $S$ be a quantum stopping time.

(i) $M_{\breve{S}}=E_{S} M_{\breve{S}} E_{S}$.

(ii) $\check{\pi}\left(M_{\widetilde{S \wedge t}}\right)_{t}=M_{\widetilde{S \wedge t}}$ for all $t \in[0, \infty]$.

(iii) The process $M^{\breve{S}}:=\left(M_{\widetilde{S \wedge t}}\right)_{t \in \mathbb{R}_{+}}$is a vacuum-adapted martingale closed by $M_{\breve{S}}$ and such that $M_{t}^{\check{S}}=E_{S \wedge t} M_{\breve{S}} E_{S \wedge t}$ for all $t \in \mathbb{R}_{+}$. 
(iv) $S([0, t]) M_{\breve{S}} S([0, t])=S([0, t]) M_{\widetilde{S \wedge t}} S([0, t])$ for all $t \in[0, \infty]$.

Proof. The first claim is immediate, because $E_{S}$ is idempotent. Furthermore, if $t \in \mathbb{R}_{+}$then

$$
E_{t} M_{\widetilde{S \wedge t}} E_{t}=E_{t} E_{S \wedge t} M_{\infty} E_{S \wedge t} E_{t}=E_{S \wedge t} M_{\infty} E_{S \wedge t}=M_{\overparen{S \wedge t}}
$$

by Theorems 3.11(ii) and 2.11, Theorem 3.11(ii) also implies that

$$
E_{t} M_{\breve{S}} E_{t}=E_{t} E_{S} M_{\infty} E_{S} E_{t}=E_{S \wedge t} M_{\infty} E_{S \wedge t}=M_{\widetilde{S \wedge t}}=M_{t}^{\breve{S}}
$$

so $M^{\breve{S}}$ is a vacuum-adapted martingale closed by $M_{\breve{S}}$, by Proposition 5.2 . If $t \in[0, \infty]$ then

$$
E_{S \wedge t} M_{\breve{S}} E_{S \wedge t}=E_{S \wedge t} E_{S} M_{\infty} E_{S} E_{S \wedge t}=E_{S \wedge t} M_{\infty} E_{S \wedge t}=M_{\widetilde{S \wedge t}},
$$

by Theorems 2.11 and 3.11(i), so (iii) holds. Finally, Proposition 3.12 implies that

$$
S([0, t]) E_{S} M_{\infty} E_{S} S([0, t])=S([0, t]) E_{S \wedge t} M_{\infty} E_{S \wedge t} S([0, t]) .
$$

Proposition 7.3. If $M$ is a martingale closed by $M_{\infty}$ then $\mathbb{E}_{\Omega}\left[M_{\breve{T}}\right]=\mathbb{E}_{\Omega}\left[M_{0}\right]$ for any quantum stopping time $T$, where $\mathbb{E}_{\Omega}$ is the vacuum state (4.1).

Proof. Note that

$$
\mathbb{E}_{\Omega}\left[M_{\breve{T}}\right]=\left\langle\varepsilon(0), E_{T} M_{\infty} E_{T} \varepsilon(0)\right\rangle=\left\langle\varepsilon(0), E_{0} E_{T} M_{\infty} E_{T} E_{0} \varepsilon(0)\right\rangle=\left\langle\varepsilon(0), M_{0} \varepsilon(0)\right\rangle=\mathbb{E}_{\Omega}\left[M_{0}\right] .
$$

Theorem 7.4 (Optional Sampling). Let $M$ be a closed martingale and let $S$ and $T$ be quantum stopping times with $S \leqslant T$. Then

$$
\left(M_{\breve{S}}\right)_{\breve{T}}=M_{\breve{S}}=\left(M_{\breve{T}}\right)_{\breve{S}}
$$

Proof. Suppose $M$ is closed by $M_{\infty}$. Theorem 3.11 (i) implies that

$$
\left(M_{\breve{T}}\right)_{\breve{S}}=E_{S} E_{T} M_{\infty} E_{T} E_{S}=E_{S} M_{\infty} E_{S}=M_{\breve{S}}
$$

and

$$
\left(M_{\breve{S}}\right)_{\breve{T}}=E_{T} E_{S} M_{\infty} E_{S} E_{T}=E_{S} M_{\infty} E_{S}=M_{\breve{S}}
$$

Theorem 6.14 motivates the definition in the following theorem, which was established by Coquio [19, Theorem 3.5]; the proof given here is a shortening of hers.

Theorem 7.5. Let $Z \in B(\mathcal{F})$ and let $S$ be a quantum stopping time. Then

$$
Z_{\widehat{S}}:=Z_{\breve{S}}+\int_{0}^{\infty} I_{\mathrm{k}} \otimes S([0, s]) \widehat{\pi}\left(Z_{\breve{S}}\right)_{s} S([0, s]) \mathrm{d} \Lambda_{s}
$$

extends to an element of $B(\mathcal{F})$, denoted the same way, with $\left\|Z_{\widehat{S}}\right\| \leqslant\|Z\|$. 
Proof. Suppose first that $S$ is discrete and let $Y$ denote the integral on the right-hand side of (7.1). From Proposition 6.6 and the remark at the end of Definition 6.4, it follows that

$$
Z_{\breve{S}}=E_{S} Z_{\widehat{S}}=E_{S} Z_{\breve{S}}+E_{S} Y
$$

so $E_{S} Y=0$ and

$$
\widehat{\pi}\left(E_{S}\right)_{t} \widehat{\pi}(Y)_{t}=\widehat{\pi}\left(E_{S}\right)_{t} \int_{0}^{t} I_{\mathrm{k}} \otimes S([0, s]) \widehat{\pi}\left(Z_{\breve{S}}\right)_{s} S([0, s]) \mathrm{d} \Lambda_{s}=0
$$

for all $t \in[0, \infty]$. Thus if $\theta \in \mathcal{E}$ then, as $S([0, s])$ commutes with $\widehat{\pi}\left(E_{S}\right)_{s}$ for all $s \in[0, \infty]$, the weak form of the quantum Itô product formula for gauge integrals, Theorem A.4, implies that

$$
\begin{aligned}
\left\|Z_{\widehat{S}} \theta\right\|^{2}-\left\|Z_{\breve{S}} \theta\right\|^{2} & =\left\|\left(Z_{\widehat{S}}-Z_{\breve{S}}\right) \theta\right\|^{2} \\
& =\int_{0}^{\infty}\left\|\left(I_{\mathrm{k}} \otimes S([0, s]) \widehat{\pi}\left(Z_{\breve{S}}\right)_{s} S([0, s])\right) \nabla_{s} \theta\right\|^{2} \mathrm{~d} s \\
& \leqslant\|Z\|^{2} \int_{0}^{\infty}\left\|\left(I_{\mathrm{k}} \otimes S([0, s]) \widehat{\pi}\left(E_{S}\right)_{s} S([0, s])\right) \nabla_{s} \theta\right\|^{2} \mathrm{~d} s \\
& =\|Z\|^{2}\left(\|\theta\|^{2}-\left\|E_{S} \theta\right\|^{2}\right),
\end{aligned}
$$

where

$$
\nabla: \mathcal{E} \rightarrow L^{2}\left(\mathbb{R}_{+} ; \mathbf{k} \otimes \mathcal{F}\right) ; \quad(\nabla \varepsilon(f))(t)=\nabla_{t} \varepsilon(f):=f(t) \varepsilon(f)
$$

is the linear gradient operator; the final identity follows from the first line by taking $Z=I$. Hence

$$
\left\|Z_{\widehat{S}} \theta\right\|^{2} \leqslant\|Z\|^{2}\left(\left\|E_{S} \theta\right\|^{2}+\|\theta\|^{2}-\left\|E_{S} \theta\right\|^{2}\right)=\|Z\|^{2}\|\theta\|^{2},
$$

and $Z_{\widehat{S}}$ extends as claimed.

For a general quantum stopping time $S$, let $S_{n}$ be a sequence of discrete quantum stopping times such that $S_{n} \Rightarrow S$. Then $Z_{\widetilde{S_{n}}} \rightarrow Z_{\breve{S}}$ in the strong operator topology and

$$
\begin{aligned}
\int_{0}^{\infty} I_{\mathrm{k}} \otimes S_{n}([0, s]) \widehat{\pi}\left(Z_{\widetilde{S_{n}}}\right)_{s} S_{n}([0, s]) \mathrm{d} \Lambda_{s} & \\
& \rightarrow \int_{0}^{\infty} I_{\mathrm{k}} \otimes S([0, s]) \widehat{\pi}\left(Z_{\breve{S}}\right)_{s} S([0, s]) \mathrm{d} \Lambda_{s}
\end{aligned}
$$

in the strong operator topology on $\mathcal{E}$, by Lemma A.7. Hence $\left\|Z_{\breve{S}} \theta\right\| \leqslant\|Z\|\|\theta\|$ for all $\theta \in \mathcal{E}$ and the result follows.

Remark 7.6. It is readily verified that $(Z, S) \mapsto Z_{\widehat{S}}$ is jointly continuous on the product of any bounded subset of $B(\mathcal{F})$ with the collection of all quantum stopping times, when $B(\mathcal{F})$ is equipped with the strong operator topology and the collection of all quantum stopping times is given the topology of Remark 3.8 . 
Consequently,

$$
Z_{\widehat{S}}=Z_{\breve{S}}+\int_{0}^{\infty} I_{\mathrm{k}} \otimes S([0, s]) \check{\pi}\left(Z_{\widehat{S}}\right)_{s} S([0, s]) \mathrm{d} \Lambda_{s}
$$

for any $Z \in B(\mathcal{F})$ and any quantum stopping time $S$, since this identity agrees with (6.2) when $S$ is discrete and extends to the general case by approximation.

Furthermore, as $I_{\widehat{T}}=I$ for any discrete quantum stopping time $T$, so

$$
\begin{aligned}
I=I_{\widehat{S}}=E_{S}+\int_{0}^{\infty} I_{\mathrm{k}} \otimes S([0, s]) \widehat{\pi}\left(E_{S}\right)_{s} \mathrm{~d} \Lambda_{s} & \\
& \Longleftrightarrow E_{S}=I-\int_{0}^{\infty} I_{\mathrm{k}} \otimes S([0, s]) \widehat{\pi}\left(E_{S}\right)_{s} \mathrm{~d} \Lambda_{s}
\end{aligned}
$$

for any quantum stopping time $S$. This identity, which is believed to be novel, expresses the time projection $E_{S}$ using an identity-adapted gauge integral; it should be compared with the first identity in (3.5).

The following result [19, Proposition 3.11] is the identity-adapted counterpart of Theorem [3.6]

Proposition 7.7. Let $S$ be a quantum stopping time. If $t \in[0, \infty]$ then

$$
\widehat{\pi}\left(E_{S}\right)_{t}=I-\int_{0}^{t} I_{\mathrm{k}} \otimes S([0, s]) \widehat{\pi}\left(E_{S}\right)_{s} \mathrm{~d} \Lambda_{s}=E_{S}+\int_{t}^{\infty} I_{\mathrm{k}} \otimes S([0, s]) \widehat{\pi}\left(E_{S}\right)_{s} \mathrm{~d} \Lambda_{s} .
$$

Proof. The first identity follows from (7.2) and the fact that the gauge integral of an identityadapted process is an identity-adapted martingale. Alternatively, applying Theorem A.8 to (3.5) gives that

$$
\widehat{\pi}\left(E_{S}\right)_{t}-I=\widehat{\pi}\left(E_{S}-I\right)_{t}=-\int_{0}^{t} I_{\mathrm{k}} \otimes\left(S([0, s])+\widehat{\pi}\left(E_{S}-I\right)_{s}\right) \mathrm{d} \Lambda_{s}
$$

so

$$
\widehat{\pi}\left(E_{S}\right)_{t}=I-\int_{0}^{t} I_{\mathrm{k}} \otimes\left(S([0, s]) \widehat{\pi}\left(E_{S}\right)_{s}+S((s, \infty])\left(I-\widehat{\pi}\left(E_{S}\right)_{s}\right)\right) \mathrm{d} \Lambda_{s} .
$$

This gives the first claim, since Theorem 3.11 (iv) and Proposition 3.12 imply that

$$
S((s, \infty]) \widehat{\pi}\left(E_{S}\right)_{s}=S((s, \infty]) \widehat{\pi}\left(E_{S \wedge s}\right)_{s}=S((s, \infty]) \widehat{\pi}\left(E_{s}\right)_{s}=S((s, \infty]) .
$$

For the second, note that the first claim with $t=\infty$ yields the identity

$$
E_{S}+\int_{t}^{\infty} I_{\mathrm{k}} \otimes S([0, s]) \widehat{\pi}\left(E_{S}\right)_{s} \mathrm{~d} \Lambda_{s}=I-\int_{0}^{t} I_{\mathrm{k}} \otimes S([0, s]) \widehat{\pi}\left(E_{S}\right)_{s} \mathrm{~d} \Lambda_{s}
$$

Proposition 7.8. If $Z \in B(\mathcal{F})$ and $S$ is a quantum stopping time then

$$
\left(Z_{\breve{S}}\right)_{\breve{S}}=Z_{\breve{S}}, \quad\left(Z_{\widehat{S}}\right)_{\breve{S}}=Z_{\breve{S}}, \quad\left(Z_{\breve{S}}\right)_{\widehat{S}}=Z_{\widehat{S}} \quad \text { and } \quad\left(Z_{\widehat{S}}\right)_{\widehat{S}}=Z_{\widehat{S}} .
$$

Furthermore, $E_{S} Z_{\widehat{S}}=Z_{\breve{S}}$. 
Proof. The first identity is trivial, and the third follows immediately from it. The fifth is true if $S$ is discrete, as noted in the proof of Theorem 7.5, so holds in general by approximation. The second is now immediate, and the fourth identity follows from the third and the second.

Remark 7.9. If $Z, W \in B(\mathcal{F})$ and $S$ is a quantum stopping time then working as in the proof of Theorem 7.5 gives that

$$
\left(Z_{\widehat{S}} W\right)_{\widehat{S}}-Z_{\widehat{S}} W_{\widehat{S}}=\int_{0}^{\infty} I_{\mathrm{k}} \otimes S([0, s]) \widehat{\pi}\left(Z_{\breve{S}}\right)_{s} S((s, \infty]) \widehat{\pi}\left(W_{\breve{S}}\right)_{s} S([0, s]) \mathrm{d} \Lambda_{s} .
$$

(Cf. the formula given in [19, Remark 3.13].) Thus, as noted by Coquio, the map $Z \mapsto Z_{\widehat{S}}$ is not, in general, a conditional expectation on $B(\mathcal{F})$, in contrast to vacuum-adapted stopping: see Proposition 4.2 .

Definition 7.10 ([19, Theorem 3.5]). The result of identity-adapted stopping a martingale $M$ closed by $M_{\infty}$ at a quantum stopping time $S$ is

$$
M_{\widehat{S}}:=\left(M_{\infty}\right)_{\widehat{S}}=M_{\breve{S}}+\int_{0}^{\infty} I_{\mathrm{k}} \otimes S([0, s]) \widehat{\pi}\left(M_{\breve{S}}\right)_{s} S([0, s]) \mathrm{d} \Lambda_{s} .
$$

Theorem 7.11. If $S$ is a quantum stopping time and $M$ is a martingale then

$$
\check{\pi}\left(M_{\breve{S}}\right)_{t}=M_{\widetilde{S \wedge t}} \quad \text { and } \quad \widehat{\pi}\left(M_{\widehat{S}}\right)_{t}=M_{\widehat{S \wedge t}} \quad \text { for all } t \in[0, \infty] ;
$$

in particular, the processes $M^{\breve{S}}$ and $M^{\widehat{S}}$ are martingales closed by $M_{\breve{S}}$.

Proof. The first identity is an immediate consequence of Theorem 3.11 (iv), that $E_{t} E_{S}=E_{S \wedge t}$. The second was established by Coquio when $S$ is discrete [19, Properties 3.3(3)] and holds in general by an approximation argument. The final remark follows from Propositions 5.2 and 5.3 .

The following result is due to Coquio [19, Proposition 3.9].

Theorem 7.12 (Optional Sampling). Let $M$ be a closed martingale and let $S$ and $T$ be quantum stopping times with $S \leqslant T$. Then

$$
\left(M_{\widehat{T}}\right)_{\widehat{S}}=M_{\widehat{S}} .
$$

Proof. Note first that, since $E_{S} E_{T}=E_{S}$, so

$$
\left(M_{\widehat{T}}\right)_{\breve{S}}=E_{S} M_{\widehat{T}} E_{S}=E_{S} E_{T} M_{\widehat{T}} E_{S}=E_{S} M_{\breve{T}} E_{S}=M_{\breve{S}},
$$

where the penultimate equality follows from Proposition 7.8. Hence

$$
\begin{aligned}
\left(M_{\widehat{T}}\right)_{\widehat{S}} & =\left(M_{\widehat{T}}\right)_{\breve{S}}+\int_{0}^{\infty} I_{\mathrm{k}} \otimes S([0, s]) \widehat{\pi}\left(\left(M_{\widehat{T}}\right)_{\breve{S}}\right)_{s} S([0, s]) \mathrm{d} \Lambda_{s} \\
& =M_{\breve{S}}+\int_{0}^{\infty} I_{\mathrm{k}} \otimes S([0, s]) \widehat{\pi}\left(M_{\breve{S}}\right)_{s} S([0, s]) \mathrm{d} \Lambda_{s} \\
& =M_{\widehat{S}} .
\end{aligned}
$$


Question 7.13. Suppose $S$ and $T$ are quantum stopping times, with $S \leqslant T$. If $Z \in B(\mathcal{F})$ then

$$
\left(Z_{\breve{S}}\right)_{\breve{T}}=\left(Z_{\breve{T}}\right)_{\breve{S}}=Z_{\breve{S}}=\left(Z_{\widehat{T}}\right)_{\breve{S}} \text {, }
$$

by Theorem 3.11 and the proof of Theorem 7.12 , whereas

$$
\left(Z_{\breve{T}}\right)_{\widehat{S}}=Z_{\widehat{S}}=\left(Z_{\widehat{T}}\right)_{\widehat{S}},
$$

by the second identity in (7.4) and Theorem 7.12, What are

$$
\left(Z_{\widehat{S}}\right)_{\breve{T}}, \quad\left(Z_{\breve{S}}\right)_{\widehat{T}} \quad \text { and } \quad\left(Z_{\widehat{S}}\right)_{\widehat{T}} ?
$$

\section{$8 \quad$ Stopping closed FV processes}

Definition 8.1. The process $Y=\left(Y_{t}\right)_{t \in \mathbb{R}_{+}}$is said to be an $F V$ process if there exists an integrand process $H$ such that $s \mapsto H_{s} x$ is strongly measurable for all $x \in \mathcal{F}$ and $\|H\|: s \mapsto\left\|H_{s}\right\|$ is locally integrable, so that the integral

$$
Y_{t}=\int_{0}^{t} H_{s} \mathrm{~d} s
$$

exists pointwise as a Bochner integral for all $t \in \mathbb{R}_{+}$; we write $Y=\int H \mathrm{~d} t$ to denote this. If $\|H\|$ is integrable then $Y_{\infty}:=\int_{0}^{\infty} H_{t} \mathrm{~d} t$ exists pointwise and the $\mathrm{FV}$ process $Y$ is closed by $Y_{\infty}$. The sets of FV processes and closed FV processes are subalgebras of the algebra of processes.

If the integrand process $H$ is identity adapted or vacuum adapted then $Y$ has the same property.

Definition 8.2. If $Y=\int H \mathrm{~d} t$ is an FV process which is closed by $Y_{\infty}$ and the discrete quantum stopping time $T$ has support $\left\{t_{1}<\cdots<t_{n}\right\}$ then, letting $T_{i}:=T\left(\left\{t_{i}\right\}\right)$ as usual,

$$
\begin{aligned}
Y_{\breve{T}} & =\sum_{i, j=1}^{n} T_{i} E_{t_{i}} Y_{t_{i} \vee t_{j}} E_{t_{j}} T_{j} \\
& =E_{T} Y_{\infty} E_{T}-\sum_{i, j=1}^{n} \int_{t_{i} \vee t_{j}}^{\infty} T_{i} E_{t_{i}} H_{s} E_{t_{j}} T_{j} \mathrm{~d} s \\
& =E_{T} Y_{\infty} E_{T}-\int_{0}^{\infty} \sum_{i, j=1}^{n} 1_{\left[t_{i}, \infty\right)}(s) T_{i} E_{t_{i}} H_{s} 1_{\left[t_{j}, \infty\right)}(s) E_{t_{j}} T_{j} \mathrm{~d} s \\
& =E_{T} Y_{\infty} E_{T}-E_{T} \int_{0}^{\infty} T([0, s]) H_{s} T([0, s]) \mathrm{d} s E_{T} .
\end{aligned}
$$

Hence, for any quantum stopping time $S$ we let

$$
\begin{aligned}
Y_{\breve{S}} & :=E_{S}\left(Y_{\infty}-\int_{0}^{\infty} S([0, s]) H_{s} S([0, s]) \mathrm{d} s\right) E_{S} \\
& =\int_{0}^{\infty} E_{S}\left(H_{s}-S([0, s]) H_{s} S([0, s])\right) E_{S} \mathrm{~d} s .
\end{aligned}
$$


Note that $E_{S} Y_{\breve{S}}=Y_{\breve{S}}=Y_{\breve{S}} E_{S}$, and that $S \mapsto Y_{\breve{S}}$ is continuous when the collection of quantum stopping times has the topology described in Remark 3.8 and $B(\mathcal{F})$ is equipped with the strong operator topology.

Remark 8.3. If $S$ corresponds to a classical stopping time $\tau$ and $Y=\int H \mathrm{~d} t$ is a classical $\mathrm{FV}$ process, for any $\omega$ in the underlying sample space,

$$
\begin{aligned}
\int_{0}^{\infty}\left(H_{s}-S([0, s]) H_{s} S([0, s])\right)(\omega) \mathrm{d} s & =\int_{0}^{\infty}\left(S((s, \infty]) H_{s}\right)(\omega) \mathrm{d} s \\
& =\int_{0}^{\tau(\omega)} H_{s}(\omega) \mathrm{d} s \\
& =\left(Y_{\tau}\right)(\omega) .
\end{aligned}
$$

Proposition 8.4. If $S$ is a quantum stopping time, $Y$ is an $F V$ process which is closed by $Y_{\infty}$ and $t \in[0, \infty]$ then

$$
S([0, t]) Y_{\breve{S}} S([0, t])=S([0, t]) Y_{\widetilde{S \wedge t}} S([0, t]) .
$$

Proof. Note that, by the definition of $Y_{\breve{S}}$ and Proposition 3.12 ,

$$
\begin{aligned}
S([0, t]) Y_{\breve{S}} S([0, t])= & S([0, t]) E_{S \wedge t} Y_{\infty} E_{S \wedge t} S([0, t]) \\
& -E_{S \wedge t} \int_{0}^{\infty} S([0, s \wedge t]) H_{s} S([0, s \wedge t]) \mathrm{d} s E_{S \wedge t} \\
= & S([0, t]) Y_{S \wedge t} S([0, t]),
\end{aligned}
$$

since $S([0, s \wedge t])=S([0, t])(S \wedge t)([0, s])$ for all $s \in \mathbb{R}_{+}$.

Definition 8.5. A semimartingale is a process of the form $X=M+Y$, where $M$ is a martingale closed by $M_{\infty}$ and $Y=\int H \mathrm{~d} t$ is a $\mathrm{FV}$ process closed by $Y_{\infty}$, with the integrand process $H$ is identity or vacuum adapted.

(Strictly speaking, this is a closed semimartingale, but no other sort of semimartingale will be considered.)

For any quantum stopping time $S$, the stopped semimartingale

$$
\begin{aligned}
X_{\breve{S}}: & =M_{\breve{S}}+Y_{\breve{S}} \\
& =E_{S}\left(M_{\infty}+Y_{\infty}\right) E_{S}-\int_{0}^{\infty} E_{S} S([0, s]) H_{s} S([0, s]) E_{S} \mathrm{~d} s .
\end{aligned}
$$

The following lemma shows that the decomposition $X=M+Y$ is unique; it follows that (8.1) is a good definition.

Lemma 8.6. Let $M$ be a martingale, let $Y=\int H \mathrm{~d} t$ be an $F V$ process and suppose the integrand process $H$ is either identity adapted or vacuum adapted. If $M+Y=0$ then $M=0$ and $Y=0$. 
Proof. It suffices to prove that $Y=0$. To see this, first recall that $M$ is a martingale if and only if $E_{s} M_{t} E_{s}=M_{s} E_{s}=E_{s} M_{s}$ for all $s, t \in \mathbb{R}_{+}$such that $s \leqslant t$. Then, for such $s$ and $t$,

$$
0=E_{s}\left(M_{t}+Y_{t}-M_{s}-Y_{s}\right) E_{s}=E_{s}\left(Y_{t}-Y_{s}\right) E_{s}=\int_{s}^{t} E_{s} H_{r} E_{s} \mathrm{~d} r
$$

given $x \in \mathcal{F}$ it follows that $\check{\pi}(H)_{s} x=E_{s} H_{s} E_{s} x=0$ for almost all $s \in \mathbb{R}_{+}$, so $s \mapsto H_{s} x=0$ almost everywhere and the claim follows.

The next result was observed by Coquio [19, Proof of Proposition 3.15].

Lemma 8.7. Let the $F V$ process $Y=\int H \mathrm{~d} t$ be closed by $Y_{\infty}$. Then

$$
\widehat{\pi}(Y)_{t}=E_{t} Y_{\infty} E_{t}+\int_{t}^{\infty} I_{\mathrm{k}} \otimes \widehat{\pi}\left(E_{t} Y_{s} E_{t}\right)_{s} \mathrm{~d} \Lambda_{s}-\int_{t}^{\infty} \widehat{\pi}\left(E_{t} H_{s} E_{t}\right)_{s} \mathrm{~d} s
$$

for all $t \in[0, \infty]$.

Proof. If $f, g \in L^{2}\left(\mathbb{R}_{+} ; \mathrm{k}\right)$ then

$$
\begin{aligned}
&\langle\varepsilon(f),\left.\int_{t}^{\infty} I_{\mathrm{k}} \otimes \widehat{\pi}\left(E_{t} Y_{s} E_{t}\right)_{s} \mathrm{~d} \Lambda_{s} \varepsilon(g)\right\rangle \\
&= \int_{t}^{\infty}\langle f(s), g(s)\rangle\left\langle\varepsilon\left(1_{[0, t)} f\right), Y_{s} \varepsilon\left(1_{[0, t)} g\right)\right\rangle\left\langle\varepsilon\left(1_{[s, \infty)} f\right), \varepsilon\left(1_{[s, \infty)} g\right)\right\rangle \mathrm{d} s \\
&=- {\left.\left[\varepsilon\left(1_{[0, t)} f\right), Y_{s} \varepsilon\left(1_{[0, t)} g\right)\right\rangle\left\langle\varepsilon\left(1_{[s, \infty)} f\right), \varepsilon\left(1_{[s, \infty)} g\right)\right\rangle\right]_{t}^{\infty} } \\
& \quad+\int_{t}^{\infty}\left\langle\varepsilon\left(1_{[0, t)} f\right), H_{s} \varepsilon\left(1_{[0, t)} g\right)\right\rangle\left\langle\varepsilon\left(1_{[s, \infty)} f\right), \varepsilon\left(1_{[s, \infty)} g\right)\right\rangle \mathrm{d} s \\
&=\left\langle\varepsilon(f),\left(\widehat{\pi}(Y)_{t}-E_{t} Y_{\infty} E_{t}\right) \varepsilon(g)\right\rangle+\left\langle\varepsilon(f), \int_{t}^{\infty} \widehat{\pi}\left(E_{t} H_{s} E_{t}\right)_{s} \mathrm{~d} s \varepsilon(g)\right\rangle .
\end{aligned}
$$

Definition 8.8. It follows from Definition 6.2 and Lemma 8.7 that if $T$ is a discrete quantum stopping time and the FV process $Y=\int H \mathrm{~d} t$ is closed by $Y_{\infty}$ then

$$
\begin{aligned}
Y_{\widehat{T}}=E_{T} Y_{\infty} E_{T}+\int_{0}^{\infty} I_{\mathrm{k}} \otimes T([0, s]) \widehat{\pi}\left(E_{T} Y_{s} E_{T}\right)_{s} T([0, s]) \mathrm{d} \Lambda_{s} \\
-\int_{0}^{\infty} T([0, s]) \widehat{\pi}\left(E_{T} H_{s} E_{T}\right)_{s} T([0, s]) \mathrm{d} s
\end{aligned}
$$

The identity (8.2) is used by Coquio [19, Proposition 3.15] to motivate the following definition: if $S$ is a quantum stopping time and $X=M+Y$ is a semimartingale then

$$
\begin{aligned}
& X_{\widehat{S}}:=M_{\widehat{S}}+Y_{\widehat{S}} \\
&=E_{S}\left(M_{\infty}+Y_{\infty}\right) E_{S}+\int_{0}^{\infty} I_{\mathrm{k}} \otimes S([0, s]) \widehat{\pi}\left(E_{S} X_{s} E_{S}\right)_{s} S([0, s]) \mathrm{d} \Lambda_{s} \\
& \quad-\int_{0}^{\infty} S([0, s]) \widehat{\pi}\left(E_{S} H_{s} E_{S}\right)_{s} S([0, s]) \mathrm{d} s .
\end{aligned}
$$


(Recall that $\widehat{\pi}(M)_{s}=\widehat{\pi}\left(M_{\infty}\right)_{s}$ for all $s \in \mathbb{R}_{+}$, by the martingale property.)

Note that $S \mapsto Y_{S}$ is continuous when $B(\mathcal{F})$ is equipped with the strong operator topology and the set of quantum stopping times has the topology defined in Remark 3.8 ,

Remark 8.9. Let $X=M+Y$ be a semimartingale. Proposition 6.6 gives that $E_{S} X_{\widehat{S}}=$ $X_{\breve{S}}$ when $S$ is discrete, and therefore this identity holds in general by approximation. As $E_{S} S([0, s])=S([0, s]) E_{S}=S([0, s]) E_{S} E_{s}$ for all $s \in[0, \infty]$, by Proposition [3.12, so

$$
E_{S} \int_{0}^{\infty} S([0, s]) \widehat{\pi}\left(E_{S} H_{s} E_{S}\right)_{s} S([0, s]) \mathrm{d} s=\int_{0}^{\infty} S([0, s]) E_{S} H_{s} E_{S} S([0, s]) \mathrm{d} s .
$$

Comparing (8.1) with $E_{S} X_{\widehat{S}}$, it follows that

$$
E_{S} \int_{0}^{\infty} I_{\mathrm{k}} \otimes S([0, s]) \widehat{\pi}\left(E_{S} X_{s} E_{S}\right)_{s} S([0, s]) \mathrm{d} \Lambda_{s}=0
$$

and the gauge integral can again be seen as an artifact produced by identity adaptedness.

\section{$9 \quad$ Stopping regular semimartingales}

For the terminology used throughout this section, see the appendix, Section A.

Example 9.1. Recall that, for any $f \in L^{2}\left(\mathbb{R}_{+} ; \mathrm{k}\right)$, the Weyl operator $W(f)$ is the unitary operator on $\mathcal{F}$ such that

$$
W(f) \varepsilon(g)=\exp \left(-\frac{1}{2}\|f\|^{2}-\langle f, g\rangle\right) \varepsilon(g+f) \quad \text { for all } g \in L^{2}\left(\mathbb{R}_{+} ; \mathbf{k}\right) .
$$

Setting

$$
W(f)_{t}:=W\left(1_{[0, t)} f\right)=\widehat{\pi}(W(f))_{t} \quad \text { and } \quad V(f)_{t}:=E_{t} W\left(1_{[0, t)} f\right)=\check{\pi}(W(f))_{t}
$$

produces identity-adapted and vacuum-adapted regular semimartingales, with

$$
\begin{aligned}
W(f)_{t}=-\int_{0}^{t}\left\langle f(s)\left|\otimes W(f)_{s} \mathrm{~d} A_{s}+\int_{0}^{t}\right| f(s)\right\rangle & \otimes W(f)_{s} \mathrm{~d} A_{s} \\
& -\frac{1}{2} \int_{0}^{t}\|f(s)\|^{2} W(f)_{s} \mathrm{~d} s
\end{aligned}
$$

and $\quad V(f)_{t}=\int_{0}^{t} I_{\mathrm{k}} \otimes V(f)_{s} \mathrm{~d} \Lambda_{s}-\int_{0}^{t}\langle f(s)| \otimes V(f)_{s} \mathrm{~d} A_{s}$

$$
+\int_{0}^{t}|f(s)\rangle \otimes V(f)_{s} \mathrm{~d} A_{s}-\frac{1}{2} \int_{0}^{t}\|f(s)\|^{2} V(f)_{s} \mathrm{~d} s
$$

for all $t \in[0, \infty]$; the operator $\langle x| \in B(\mathrm{k} ; \mathbb{C})$ is such that $\langle x| y=\langle x, y\rangle$ for all $x, y \in \mathrm{k}$, and its adjoint $|x\rangle \in B(\mathbb{C} ; \mathrm{k})$ is such that $|x\rangle \lambda=\lambda x$ for all $\lambda \in \mathbb{C}$ and $x \in \mathrm{k}$. (Cf. Theorem A.8.) 
The class of regular $\Omega$-semimartingales is closed under vacuum-adapted stopping; the following result gives this in explicit form. Coquio obtained an analogous result for regular quantum semimartingales [19, Proposition 3.16].

Theorem 9.2. Let the regular $\Omega$-semimartingale $X=M+Y$ have martingale part $M=$ $\int N \mathrm{~d} \Lambda+P \mathrm{~d} A+Q \mathrm{~d} A^{\dagger}$ and $F V$ part $Y=\int R \mathrm{~d} t$. If $S$ is a quantum stopping time then

$$
X_{\breve{S}}=\int_{0}^{\infty} \check{S}_{N_{s}} \mathrm{~d} \Lambda_{s}+\int_{0}^{\infty} \check{S}_{P_{s}} \mathrm{~d} A_{s}+\int_{0}^{\infty} \check{S}_{Q_{s}} \mathrm{~d} A_{s}^{\dagger}+\int_{0}^{\infty} \check{S}_{R_{s}} \mathrm{~d} s
$$

where, for all $s \in \mathbb{R}_{+}$,

$$
\begin{aligned}
& \breve{S}_{N_{s}}:=\left(I_{\mathrm{k}} \otimes S((s, \infty])\right) N_{s}\left(I_{\mathrm{k}} \otimes S((s, \infty])\right), \\
& \check{S}_{P_{s}}:=E_{S} P_{s}\left(I_{\mathrm{k}} \otimes S((s, \infty])\right) \\
& \check{S}_{Q_{s}}:=\left(I_{\mathrm{k}} \otimes S((s, \infty])\right) Q_{s} E_{S} \\
& \text { and } \quad \check{S}_{R_{s}}:=E_{S} R_{s} E_{S}-S([0, s]) E_{S} R_{s} E_{S} S([0, s]) .
\end{aligned}
$$

Proof. If $X=\int N \mathrm{~d} \Lambda+P \mathrm{~d} A+Q \mathrm{~d} A^{\dagger}+R \mathrm{~d} t$ and $X^{\prime}=\int N^{\prime} \mathrm{d} \Lambda$ are regular $\Omega$-semimartingales then Theorem $A .2$ implies that

$$
\begin{aligned}
& X X^{\prime}=\int N N^{\prime} \mathrm{d} \Lambda+P N^{\prime} \mathrm{d} A+Q X^{\prime} \mathrm{d} A^{\dagger}+R X^{\prime} \mathrm{d} t \\
& X^{\prime} X=\int N^{\prime} N \mathrm{~d} \Lambda+X^{\prime} P \mathrm{~d} A+N^{\prime} Q \mathrm{~d} A^{\dagger}+X^{\prime} R \mathrm{~d} t
\end{aligned}
$$

and $\quad X^{\prime} X X^{\prime}=\int N^{\prime} N N^{\prime} \mathrm{d} \Lambda+X^{\prime} P N^{\prime} \mathrm{d} A+N^{\prime} Q X^{\prime} \mathrm{d} A^{\dagger}+X^{\prime} R X^{\prime} \mathrm{d} t$

so

$$
\begin{aligned}
& X^{\prime} X X^{\prime}-X^{\prime} X-X X^{\prime}+X \\
& =\int\left(N^{\prime} N N^{\prime}-N^{\prime} N-N N^{\prime}+N\right) \mathrm{d} \Lambda+\left(X^{\prime} P N^{\prime}-X^{\prime} P-P N^{\prime}+P\right) \mathrm{d} A \\
& \quad+\left(N^{\prime} Q X^{\prime}-N^{\prime} Q-Q X^{\prime}+Q\right) \mathrm{d} A^{\dagger}+\left(X^{\prime} R X^{\prime}-X^{\prime} R-R X^{\prime}+R\right) \mathrm{d} t \\
& =\int\left(I_{\mathrm{k} \otimes \mathcal{F}}-N^{\prime}\right) N\left(I_{\mathrm{k} \otimes \mathcal{F}}-N^{\prime}\right) \mathrm{d} \Lambda+\left(I-X^{\prime}\right) P\left(I_{\mathrm{k} \otimes \mathcal{F}}-N^{\prime}\right) \mathrm{d} A \\
& \quad+\left(I_{\mathrm{k} \otimes \mathcal{F}}-N^{\prime}\right) Q\left(I-X^{\prime}\right) \mathrm{d} A^{\dagger}+\left(I-X^{\prime}\right) R\left(I-X^{\prime}\right) \mathrm{d} t .
\end{aligned}
$$

Taking

$$
X_{t}^{\prime}=I-E_{S \wedge t}=\int_{0}^{t} I_{\mathrm{k}} \otimes S([0, s]) E_{s} \mathrm{~d} \Lambda_{s} \quad \text { for all } t \in[0, \infty]
$$


now gives the result, since

$$
\begin{aligned}
& E_{S} X_{\infty} E_{S} \\
& =\left(X^{\prime} X X^{\prime}-X^{\prime} X-X X^{\prime}+X\right)_{\infty} \\
& =\int_{0}^{\infty}\left(I_{\mathrm{k}} \otimes S((s, \infty])\right) N_{s}\left(I_{\mathrm{k}} \otimes S((s, \infty])\right) \mathrm{d} \Lambda_{s}+E_{S} P_{s}\left(I_{\mathrm{k}} \otimes S((s, \infty])\right) \mathrm{d} A_{s} \\
& \quad+\left(I_{\mathrm{k}} \otimes S((s, \infty])\right) Q_{s} E_{S} \mathrm{~d} A_{s}^{\dagger}+E_{S} R_{s} E_{S} \mathrm{~d} s
\end{aligned}
$$

\section{Stopping the future projection}

Definition 10.1. Given $t \in \mathbb{R}_{+}$, let the isometric right shift

$$
\theta_{t}: L^{2}\left(\mathbb{R}_{+} ; \mathrm{k}\right) \rightarrow L^{2}\left(\mathbb{R}_{+} ; \mathrm{k}\right) ;\left(\theta_{t} f\right)(s):=1_{[t, \infty)}(s) f(s-t),
$$

and let $\Gamma_{t} \in B(\mathcal{F})$ be its second quantisation, so that $\Gamma_{t}^{*} \varepsilon(f)=\varepsilon(f(\cdot+t))$ for all $f \in L^{2}\left(\mathbb{R}_{+} ; \mathbf{k}\right)$. If $F_{t}:=\Gamma_{t} \Gamma_{t}^{*}$ then

$$
F_{t} \varepsilon(f)=\varepsilon\left(1_{[t, \infty)} f\right) \quad \text { for all } f \in L^{2}\left(\mathbb{R}_{+} ; \mathrm{k}\right)
$$

and $F$ is an identity-adapted martingale closed by $F_{\infty}:=E_{0}$.

Given $s, t \in[0, \infty]$ with $s \leqslant t$, note that

$$
E_{s} \Gamma_{t}=E_{0}=\Gamma_{t} E_{s} \quad \text { and } \quad E_{s} F_{t}=E_{0}=F_{t} E_{s} .
$$

(The operator $F_{t}$ is denoted by $R_{t}$ in [19, Section 4] and by $\Gamma\left(\chi_{[t, \infty)}\right)$ in [30].)

Remark 10.2. If the discrete quantum stopping time $T$ has $\left\{t_{1}<\cdots<t_{n}\right\}$ and $T_{i}:=T\left(\left\{t_{i}\right\}\right)$ for $i=1, \ldots, n$ then

$$
\Gamma_{\widehat{T}}=\sum_{i, j}^{n} T_{i} \widehat{\pi}\left(E_{t_{i}} \Gamma_{t_{i} \vee t_{j}} E_{t_{j}}\right)_{t_{i} \vee t_{j}} T_{j}=\sum_{i, j=1}^{n} T_{i} \widehat{\pi}\left(E_{0}\right)_{t_{i} \vee t_{j}} T_{j}=F_{T} F_{T}^{*},
$$

where $F_{T}:=\int_{[0, \infty]} T(\mathrm{~d} s) F_{s}$; note that $F_{s \vee t}=F_{s} F_{t}$ for all $s, t \in[0, \infty]$.

As $\Gamma$ is not a semimartingale, it is unclear how to extend this identity to hold for more general stopping times; Parthasarathy and Sinha introduced a left-stopped form of $\Gamma$ in [30, Section 5]; see also [16, Theorem 3.8].

However, similar working shows that

$$
F_{\widehat{T}}=\sum_{i, j=1}^{n} T_{i} \widehat{\pi}\left(E_{t_{i}} F_{t_{i} \vee t_{j}} E_{t_{j}}\right)_{t_{i} \vee t_{j}} T_{j}=F_{T} F_{T}^{*} .
$$

The operators $F_{T}$ and $F_{T}^{*}$ are obtained by left and right stopping the process $F$ at the quantum stopping time $T$, so it is not surprising that $F_{T} F_{T}^{*}$ corresponds to double stopping $F$. The following results shows that this intuition holds true in general. 
Lemma 10.3. The identity-adapted martingale $F$ is such that

$$
F_{t}=E_{0}+\int_{t}^{\infty} I_{\mathrm{k}} \otimes F_{s} \mathrm{~d} \Lambda_{s}=I-\int_{0}^{t} I_{\mathrm{k}} \otimes F_{s} \mathrm{~d} \Lambda_{s} \quad \text { for all } t \in[0, \infty] .
$$

Proof. Taking $M=E_{0}$ in Lemma 6.12 gives the first identity. From this it follows that

$$
I=F_{0}=E_{0}+\int_{0}^{\infty} I_{\mathrm{k}} \otimes F_{s} \mathrm{~d} \Lambda_{s}=E_{0}+\int_{0}^{t} I_{\mathrm{k}} \otimes F_{s} \mathrm{~d} \Lambda_{s}+\int_{t}^{\infty} I_{\mathrm{k}} \otimes F_{s} \mathrm{~d} \Lambda_{s},
$$

and rearranging this gives the second.

Theorem 10.4. Given an arbitrary quantum stopping time $S$, there exist a contraction $F_{S} \in$ $B(\mathcal{F})$ such that

$$
F_{S}=S(\{0\})+\text { st. } \lim _{\pi} \sum_{j=1}^{\infty} S\left(\left(\pi_{j-1}, \pi_{j}\right]\right) F_{\pi_{j}}+S(\{\infty\}) E_{0}
$$

where the limit is taken over partitions of $\mathbb{R}_{+}$, ordered by refinement. This operator $F_{S}$ is such that

$$
\left\langle F_{S} x, F_{S} y\right\rangle=\int_{[0, \infty]}\left\langle x, F_{s} y\right\rangle \mathbb{E}_{\Omega}[S(\mathrm{~d} s)] \quad \text { for all } x, y \in \mathcal{F}
$$

and

$$
F_{S}=E_{0}+\int_{0}^{\infty} I_{\mathrm{k}} \otimes S([0, s]) F_{s} \mathrm{~d} \Lambda_{s}=I-\int_{0}^{\infty} I_{\mathrm{k}} \otimes S((s, \infty]) F_{s} \mathrm{~d} \Lambda_{s} .
$$

Proof. Let $F_{S}^{\pi}:=S(\{0\})+\sum_{j=1}^{\infty} S\left(\left(\pi_{j-1}, \pi_{j}\right]\right) F_{\pi_{j}}+S(\{\infty\}) E_{0}$ and note that

$$
\left\|\left(X \otimes I_{[t}\right) F_{t} x\right\|=\|X \varepsilon(0)\|\left\|F_{t} x\right\|
$$

for all $t \in(0, \infty), X \in B\left(\mathcal{F}_{t)}\right)$ and $x \in \mathcal{F}$, so $F_{S}^{\pi}$ converges in the strong operator topology and $\left\|F_{S}^{\pi}\right\| \leqslant 1$. Let $\pi^{\prime}$ be a refinement of $\pi$, let $k_{j}$ and $l_{j}$ have the same meaning as they do in the proof of Theorem 3.3. Given $f \in L^{2}\left(\mathbb{R}_{+} ; \mathrm{k}\right)$,

$$
\begin{aligned}
\left\|\left(F_{S}^{\pi}-F_{S}^{\pi^{\prime}}\right) \varepsilon(f)\right\|^{2} & \\
& =\sum_{j=0}^{\infty} \sum_{l=1}^{l_{j}}\left\|S\left(\left(\pi_{k_{j}+l-1}^{\prime}, \pi_{k_{j}+l}^{\prime}\right]\right)\left(F_{\pi_{k_{j}+l_{j}}^{\prime}}-F_{\pi_{k_{j}+l}^{\prime}}\right) \varepsilon(f)\right\|^{2} \\
& =\sum_{j=0}^{\infty} \sum_{l=1}^{l_{j}}\left\|S\left(\left(\pi_{k_{j}+l-1}^{\prime}, \pi_{k_{j}+l}^{\prime}\right]\right) \varepsilon(0)\right\|^{2}\left\|\left(F_{\pi_{k_{j}+l_{j}}^{\prime}}-F_{\pi_{k_{j}+l}^{\prime}}\right) \varepsilon(f)\right\|^{2} .
\end{aligned}
$$

If $s, t \in \mathbb{R}_{+}$are such that $s<t$ then

$$
\left\|\left(F_{t}-F_{s}\right) \varepsilon(f)\right\|=\left\|\varepsilon\left(\left.f\right|_{[s, t)}\right)-\varepsilon\left(\left.0\right|_{[s, t)}\right)\right\|\left\|\varepsilon\left(\left.f\right|_{[t, \infty)}\right)\right\|
$$


and therefore

$$
\left\|\left(F_{S}^{\pi}-F_{S}^{\pi^{\prime}}\right) \varepsilon(f)\right\|^{2} \leqslant\|\varepsilon(f)\|^{2} \sup \left\{\exp \left(\left\|1_{\left[\pi_{j}, \pi_{j+1}\right)} f\right\|^{2}\right)-1: j \geqslant 0\right\},
$$

which tends to zero as $\pi$ is refined. Hence $F_{S} \varepsilon(f):=\lim _{\pi} F_{S}^{\pi} \varepsilon(f)$ exists for all $f \in L^{2}\left(\mathbb{R}_{+} ; \mathrm{k}\right)$; as exponential vectors are total in $\mathcal{F}$ and each $F_{S}^{\pi}$ is a contraction, the operator $F_{S}$ exists as claimed.

By (10.1), the map $s \mapsto F_{s} x$ is continuous for all $x \in \mathcal{F}$ and therefore

$$
\begin{aligned}
\left\langle F_{S} x, F_{S} y\right\rangle & =\lim _{\pi}\left\langle F_{S}^{\pi} x, F_{S}^{\pi} y\right\rangle \\
& =\langle x, S(\{0\}) y\rangle+\lim _{\pi} \sum_{j=1}^{\infty}\left\langle\varepsilon(0), S\left(\left(\pi_{j-1}, \pi_{j}\right]\right) \varepsilon(0)\right\rangle\left\langle x, F_{\pi_{j}} y\right\rangle \\
& =\int_{[0, \infty]}\left\langle x, F_{s} y\right\rangle \mathbb{E}_{\Omega}[S(\mathrm{~d} s)]
\end{aligned}
$$

for all $x, y \in \mathcal{F}$.

For the final claim, without loss of generality suppose $S(\{0\})=0$, and let $f, g \in L^{2}\left(\mathbb{R}_{+} ; \mathrm{k}\right)$, with $g$ having support in $\left[0, \pi_{n}\right]$. Then $F_{t} \varepsilon(g)=E_{0} \varepsilon(g)$ whenever $t \geqslant \pi_{n}$, so it follows that

$$
\begin{aligned}
\left\langle\varepsilon(f),\left(F_{S}^{\pi}-E_{0}\right) \varepsilon(g)\right\rangle & =\sum_{j=1}^{\infty}\left\langle\varepsilon(f), S\left(\left(\pi_{j-1}, \pi_{j}\right]\right)\left(F_{\pi_{j}}-E_{0}\right) \varepsilon(g)\right\rangle \\
& =\sum_{j=1}^{n-1} \sum_{k=j}^{n-1}\left\langle\varepsilon(f), S\left(\left(\pi_{j-1}, \pi_{j}\right]\right)\left(F_{\pi_{k}}-F_{\pi_{k+1}}\right) \varepsilon(g)\right\rangle \\
& =\sum_{k=1}^{n-1}\left\langle\varepsilon(f), \int_{\pi_{k}}^{\pi_{k+1}} I_{\mathrm{k}} \otimes S\left(\left[0, \pi_{k}\right]\right) F_{s} \mathrm{~d} \Lambda_{s} \varepsilon(g)\right\rangle,
\end{aligned}
$$

where the final equality follows from by Lemma 10.3 and Lemma A.6. The first identity is now seen to hold, since

$$
R_{\pi}^{\prime}:=\int_{0}^{\infty} \sum_{k=0}^{\infty} 1_{\left(\pi_{k}, \pi_{k+1}\right]}(s) I_{\mathrm{k}} \otimes S\left(\left(\pi_{k}, s\right]\right) F_{s} \mathrm{~d} \Lambda_{s} \varepsilon(g) \rightarrow 0
$$

as $\pi$ is refined, by Lemma A.7, working as in the proof of Theorem 3.6. The second identity is now a consequence of Lemma 10.3 .

Theorem 10.5. Let $S$ be a quantum stopping time. Then

$$
F_{S} F_{S}^{*}=E_{0}+\int_{0}^{\infty} I_{\mathrm{k}} \otimes S([0, s]) F_{s} S([0, s]) \mathrm{d} \Lambda_{s}=F_{\widehat{S}} .
$$


Proof. The second identity is immediate from (7.1) and the fact that $F$ is an identity-adapted martingale closed by $E_{0}=F_{\breve{S}}$. For the first, note first that $F_{S} E_{0}=E_{0}$ and, working as in the proof of Theorem 10.4,

$$
\int_{0}^{t} I_{\mathrm{k}} \otimes S([0, s]) F_{s} \mathrm{~d} \Lambda_{s}=S([0, t])\left(F_{S}-F_{t}\right) \quad \text { for all } t \in[0, \infty] .
$$

In particular, $S([0, t])\left(F_{S}-F_{t}\right) F_{t}=0$ and therefore

$$
\begin{aligned}
F_{S} F_{S}^{*}-E_{0}= & \left(F_{S}-E_{0}\right)\left(F_{S}-E_{0}\right)^{*} \\
= & \int_{0}^{\infty} I_{\mathrm{k}} \otimes S([0, s]) F_{s} \mathrm{~d} \Lambda_{s} \int_{0}^{\infty} I_{\mathrm{k}} \otimes F_{s} S([0, s]) \mathrm{d} \Lambda_{s} \\
= & \int_{0}^{\infty} I_{\mathrm{k}} \otimes\left(S([0, s])\left(F_{S}-F_{s}\right) F_{s} S([0, s])\right. \\
& \left.\quad+S([0, s]) F_{s}\left(F_{S}^{*}-F_{s}\right) S([0, s])+S([0, s]) F_{s} S([0, s])\right) \mathrm{d} \Lambda_{s} \\
= & \int_{0}^{\infty} I_{\mathrm{k}} \otimes S([0, s]) F_{s} S([0, s]) \mathrm{d} \Lambda_{s} .
\end{aligned}
$$

\section{A Quantum stochastic calculus}

Definition A.1. Let $\mathrm{k}_{1}$ and $\mathrm{k}_{2}$ be non-zero subspaces of $\mathrm{k}$. A $\mathrm{k}_{1}-\mathrm{k}_{2}$ process $X$ is a family of bounded operators $\left(X_{t}\right)_{t \in \mathbb{R}_{+}} \subseteq B\left(\mathrm{k}_{1} \otimes \mathcal{F} ; \mathrm{k}_{2} \otimes \mathcal{F}\right)$ such that $t \mapsto X_{t} \varepsilon(f)$ is strongly measurable for all $f \in L^{2}\left(\mathbb{R}_{+} ; \mathrm{k}\right)$.

A $\mathrm{k}_{1}-\mathrm{k}_{2}$ process $X$ is vacuum adapted if $\left(I_{\mathrm{k}_{2}} \otimes E_{t}\right) X_{t}\left(I_{\mathrm{k}_{1}} \otimes E_{t}\right)=X_{t}$ for all $t \in \mathbb{R}_{+}$.

Let $N, P, Q$ and $R$ be vacuum adapted $\mathrm{k}-\mathrm{k}, \mathrm{k}-\mathbb{C}, \mathbb{C}-\mathrm{k}$ and $\mathbb{C}-\mathbb{C}$ processes, respectively, such that

$$
\|N\|_{\infty}:=\operatorname{ess} \sup \left\{\left\|N_{t}\right\|: t \in \mathbb{R}_{+}\right\}<\infty \quad \text { and } \quad \int_{0}^{\infty}\left(\left\|P_{s}\right\|^{2}+\left\|Q_{s}\right\|^{2}+\left\|R_{s}\right\|\right) \mathrm{d} s<\infty .
$$

The gauge integral $\int N \mathrm{~d} \Lambda=\left(\int_{0}^{t} N_{s} \mathrm{~d} \Lambda_{s}\right)$, annihilation integral $\int P \mathrm{~d} A=\left(\int_{0}^{t} P_{s} \mathrm{~d} A_{s}\right)$, creation integral $\int Q \mathrm{~d} A^{\dagger}=\left(\int_{0}^{t} Q_{s} \mathrm{~d} A_{s}^{\dagger}\right)$ and time integral $\int R \mathrm{~d} t=\left(\int_{0}^{t} R_{s} \mathrm{~d} s\right)$ are the unique vacuumadapted $\mathbb{C}-\mathbb{C}$ processes such that, for all $f, g \in L^{2}\left(\mathbb{R}_{+} ; \mathrm{k}\right)$ and $t \in[0, \infty]$,

$$
\begin{aligned}
\left\langle\varepsilon(f), \int_{0}^{t} N_{s} \mathrm{~d} \Lambda_{s} \varepsilon(g)\right\rangle & =\int_{0}^{t}\left\langle f(s) \otimes \varepsilon(f), N_{s}(g(s) \otimes \varepsilon(g))\right\rangle \mathrm{d} s \\
\left\langle\varepsilon(f), \int_{0}^{t} P_{s} \mathrm{~d} A_{s} \varepsilon(g)\right\rangle & =\int_{0}^{t}\left\langle\varepsilon(f), P_{s}(g(s) \otimes \varepsilon(g))\right\rangle \mathrm{d} s \\
\left\langle\varepsilon(f), \int_{0}^{t} Q_{s} \mathrm{~d} A_{s}^{\dagger} \varepsilon(g)\right\rangle & =\int_{0}^{t}\left\langle f(s) \otimes \varepsilon(f), Q_{s} \varepsilon(g)\right\rangle \mathrm{d} s \\
\text { and } \quad\left\langle\varepsilon(f), \int_{0}^{t} R_{s} \mathrm{~d} s \varepsilon(g)\right\rangle & =\int_{0}^{t}\left\langle\varepsilon(f), R_{s} \varepsilon(g)\right\rangle \mathrm{d} s .
\end{aligned}
$$


A vacuum-adapted $\mathbb{C}-\mathbb{C}$ process $X$ is a regular $\Omega$-semimartingale if there exist vacuum-adapted processes $N, P, Q$, and $R$ as above and such that

$$
X_{t}=\int_{0}^{t} N_{s} \mathrm{~d} \Lambda_{s}+\int_{0}^{t} P_{s} \mathrm{~d} A_{s}+\int_{0}^{t} Q_{s} \mathrm{~d} A_{s}^{\dagger}+\int_{0}^{t} R_{s} \mathrm{~d} s \quad \text { for all } t \in[0, \infty]
$$

we write $X=\int N \mathrm{~d} \Lambda+P \mathrm{~d} A+Q \mathrm{~d} A^{\dagger}+R \mathrm{~d} t$ to denote this.

The martingale part of $X$ is the vacuum-adapted martingale $M=\int N \mathrm{~d} \Lambda+P \mathrm{~d} A+Q \mathrm{~d} A^{\dagger}$ which is closed by $M_{\infty}$, where

$$
M_{t}=\int_{0}^{t} N_{s} \mathrm{~d} \Lambda_{s}+\int_{0}^{t} P_{s} \mathrm{~d} A_{s}+\int_{0}^{t} Q_{s} \mathrm{~d} A_{s}^{\dagger} \quad \text { for all } t \in[0, \infty],
$$

and the $F V$ part of $X$ is the vacuum-adapted FV process $Y=\int R \mathrm{~d} t$ which is closed by

$$
Y_{\infty}=\int_{0}^{\infty} R_{s} \mathrm{~d} s
$$

Theorem A.2. Given regular $\Omega$-semimartingales $X=\int N \mathrm{~d} \Lambda+P \mathrm{~d} A+Q \mathrm{~d} A^{\dagger}+R \mathrm{~d} t$ and $X^{\prime}=$ $\int N^{\prime} \mathrm{d} \Lambda+P^{\prime} \mathrm{d} A+Q^{\prime} \mathrm{d} A^{\dagger}+R^{\prime} \mathrm{d} t$, the process $X X^{\prime}=\left(X_{t} X_{t}^{\prime}\right)_{t \in[0, \infty]}$ is a regular $\Omega$-semimartingale, with

$$
X X^{\prime}=\int N N^{\prime} \mathrm{d} \Lambda+\left(X P^{\prime}+P N^{\prime}\right) \mathrm{d} A+\left(Q X^{\prime}+N Q^{\prime}\right) \mathrm{d} A^{\dagger}+\left(X R^{\prime}+R X^{\prime}+P Q^{\prime}\right) \mathrm{d} t .
$$

Definition A.3. A $\mathrm{k}_{1}-\mathrm{k}_{2}$ process $X$ is identity adapted if and only if

$$
\left\langle\varepsilon(f), X_{t} \varepsilon(g)\right\rangle=\left\langle\varepsilon\left(1_{[0, t)} f\right), X_{t} \varepsilon\left(1_{[0, t)} g\right)\right\rangle\left\langle\varepsilon\left(1_{[t, \infty)} f\right), \varepsilon\left(1_{[t, \infty)} g\right)\right\rangle
$$

for all $f, g \in L^{2}\left(\mathbb{R}_{+} ; \mathrm{k}\right)$ and $t \in \mathbb{R}_{+}$; equivalently, $X_{t}=X_{t)} \otimes I_{[t}$, where $X_{t)} \in B\left(\mathcal{F}_{t)}\right)$, for all $t \in \mathbb{R}_{+}$.

Given a quadruple of processes $N, P, Q$ and $R$ as in Definition A.1, but identity adapted rather than vacuum adapted, there exist identity-adapted gauge, annihilation, creation and time integrals which satisfy the same inner-product identities. However, integration may not preserve boundedness in this case, other than for the time integral: the integrals will exist as linear operators with domains containing $\mathcal{E}$ such that the identities (A.1 A.4 hold. If

$$
M_{t}=\int_{0}^{t} N_{s} \mathrm{~d} \Lambda_{s}+\int_{0}^{t} P_{s} \mathrm{~d} A_{s}+\int_{0}^{t} Q_{s} \mathrm{~d} A_{s}^{\dagger}
$$

is a bounded operator for all $t \in[0, \infty]$ then the identity-adapted $\mathbb{C}-\mathbb{C}$ process $X=M+Y$, where $Y=\int H \mathrm{~d} t$, is a regular quantum semimartingale.

A weak form of Itô product formula holds for quantum semimartingales which are not necessarily regular. We only need the version for gauge integrals, which is as follows. 
Theorem A.4. Let $N$ and $N^{\prime}$ be identity-adapted $\mathrm{k}-\mathrm{k}$ processes such that $\|N\|_{\infty}<\infty$ and $\left\|N^{\prime}\right\|_{\infty}<\infty$. If $X_{t}=\int_{0}^{t} N_{s} \mathrm{~d} \Lambda_{s}$ and $X_{t}^{\prime}=\int_{0}^{t} N_{s}^{\prime} \mathrm{d} \Lambda_{s}$ then

$$
\left\langle X_{t} x, X_{t}^{\prime} x^{\prime}\right\rangle=\int_{0}^{t}\left(\left\langle\left(I_{\mathrm{k}} \otimes X_{s}\right) \nabla_{s} x, N_{s}^{\prime} \nabla_{s} x^{\prime}\right\rangle+\left\langle N_{s} \nabla_{s} x,\left(I_{\mathrm{k}} \otimes X_{s}^{\prime}\right) \nabla_{s} x^{\prime}\right\rangle+\left\langle N_{s} \nabla_{s} x, N_{s}^{\prime} \nabla_{s} x^{\prime}\right\rangle\right) \mathrm{d} s
$$

for all $t \in[0, \infty]$ and $x, x^{\prime} \in \mathcal{F}$, where

$$
\nabla: \mathcal{E} \rightarrow L^{2}\left(\mathbb{R}_{+} ; \mathbf{k} \otimes \mathcal{F}\right) ; \quad(\nabla \varepsilon(f))(t)=\nabla_{t} \varepsilon(f):=f(t) \varepsilon(f)
$$

is the linear gradient operator.

Proof. See [25, Theorem 3.15].

Remark A.5. The decomposition $X=M+Y$ is unique for both regular $\Omega$-semimartingales and regular quantum semimartingales, by Lemma 8.6. In fact, more may be shown: the quantum stochastic integrators are independent, in the sense that if

$$
\int_{0}^{\infty} N_{s} \mathrm{~d} \Lambda_{s}+\int_{0}^{\infty} P_{s} \mathrm{~d} A_{s}+\int_{0}^{\infty} Q_{s} \mathrm{~d} A_{s}^{\dagger}+\int_{0}^{\infty} R_{s} \mathrm{~d} s=0
$$

then $N_{s}=P_{s}=Q_{s}=R_{s}=0$ for almost all $s \in \mathbb{R}_{+}[24$.

Lemma A.6. Let $t \in \mathbb{R}_{+}$and $Z, W \in B\left(\mathcal{F}_{t)}\right) \otimes I_{[t}$. If $\left(N_{s}\right)_{s \in \mathbb{R}_{+}}$is a vacuum or identity-adapted $\mathrm{k}-\mathrm{k}$ process such that $\|N\|_{\infty}<\infty$ then so is $\left(1_{[t, \infty)}(s)\left(I_{\mathrm{k}} \otimes Z\right) N_{s}\left(I_{\mathrm{k}} \otimes W\right)\right)_{s \in \mathbb{R}_{+}}$, with the same type of adaptedness, and

$$
Z \int_{t}^{\infty} N_{s} \mathrm{~d} \Lambda_{s} W=\int_{t}^{\infty}\left(I_{\mathrm{k}} \otimes Z\right) N_{s}\left(I_{\mathrm{k}} \otimes W\right) \mathrm{d} \Lambda_{s}
$$

Proof. The first claim is immediate. For the second, suppose first that $Z=W(f)$, the Weyl operator corresponding to $f \in L^{2}\left(\mathbb{R}_{+} ; \mathrm{k}\right)$, as in Example 9.1. Then $Z^{*}=W(-f)$ and, if $f$ has support in $[0, t]$,

$$
\begin{aligned}
\left\langle\varepsilon(g), Z \int_{t}^{\infty} N_{s} \mathrm{~d} \Lambda_{s} \varepsilon(h)\right\rangle & \\
& =\exp \left(-\frac{1}{2}\|f\|^{2}+\langle f, g\rangle\right)\left\langle\varepsilon(g-f), \int_{t}^{\infty} N_{s} \mathrm{~d} \Lambda_{s} \varepsilon(h)\right\rangle \\
& =\exp \left(-\frac{1}{2}\|f\|^{2}+\langle f, g\rangle\right) \int_{t}^{\infty}\left\langle(g-f)(s) \otimes \varepsilon(g-f), N_{s}(h(s) \otimes \varepsilon(h))\right\rangle \mathrm{d} s \\
& =\int_{t}^{\infty}\left\langle g(s) \otimes Z^{*} \varepsilon(g), N_{s}(h(s) \otimes \varepsilon(h))\right\rangle \mathrm{d} s \\
& =\left\langle\varepsilon(g), \int_{t}^{\infty}\left(I_{\mathrm{k}} \otimes Z\right) N_{s} \mathrm{~d} \Lambda_{s} \varepsilon(h)\right\rangle
\end{aligned}
$$

for all $g, h \in L^{2}\left(\mathbb{R}_{+} ; \mathrm{k}\right)$. Since such Weyl operators are dense in $B\left(\mathcal{F}_{t)}\right) \otimes I_{[t}$ for the strong operator topology, the result holds for general $Z$ and $W=I$; the full version may be obtained by taking adjoints. 
Lemma A.7. If $N$ is a vacuum or identity-adapted $\mathrm{k}-\mathrm{k}$ process such that $\|N\|_{\infty}<\infty$ then

$$
\left\|\int_{0}^{\infty} N_{s} \mathrm{~d} \Lambda_{s} \varepsilon(f)\right\|^{2} \leqslant C_{f}^{2} \int_{0}^{\infty}\left\|N_{s}(f(s) \otimes \varepsilon(f))\right\|^{2} \mathrm{~d} s \quad \text { for all } f \in L^{2}\left(\mathbb{R}_{+} ; \mathrm{k}\right),
$$

where $C_{f}:=1$ if $N$ is vacuum adapted and $C_{f}:=\|f\|+\sqrt{1+\|f\|^{2}}$ if $N$ is identity adapted.

Proof. See [15, Proof of Theorem 18] and [25, Theorem 3.13], respectively.

Theorem A.8. If $X=\int N \mathrm{~d} \Lambda+P \mathrm{~d} A+Q \mathrm{~d} A^{\dagger}+R \mathrm{~d} t$ is a regular $\Omega$-semimartingale then $\widehat{\pi}(X)$ is a regular quantum semimartingale such that

$$
\widehat{\pi}(X)_{t}=\int_{0}^{t} \widehat{\pi}\left(N-I_{\mathrm{k}} \otimes X\right)_{s} \mathrm{~d} \Lambda_{s}+\int_{0}^{t} \widehat{\pi}(P)_{s} \mathrm{~d} A_{s}+\int_{0}^{t} \widehat{\pi}(Q)_{s} \mathrm{~d} A_{s}^{\dagger}+\int_{0}^{t} \widehat{\pi}(R)_{s} \mathrm{~d} s
$$

for all $t \in[0, \infty]$.

Proof. See [15, Corollaries 31 and 40]; the extension to a non-separable multiplicity space k is straightforward.

Notation A.9. Let $\mathrm{k}=\mathbb{C}$, let $\nu=\left(\nu_{t}\right)_{t \in \mathbb{R}_{+}}$be a standard Poisson process on the probability space $(\Omega, \mathcal{A}, \mathbb{P})$ and let $U_{P}: L^{2}(\Omega, \mathcal{A}, \mathbb{P}) \rightarrow \mathcal{F}$ be the isometric isomorphism such that the closure of $N_{t}:=\Lambda_{t}+A_{t}+A_{t}^{\dagger}+t I$ equals $U_{P} \nu_{t} U_{P}^{*}$ for all $t \in \mathbb{R}_{+}$, where $\widehat{\nu_{t}}$ acts by multiplication by $\nu_{t}$, and $U_{P} 1=\varepsilon(0)$ [23, Theorems 6.1-2]. Recall that

$$
\zeta(f):=U_{P}^{*} \varepsilon(f)=1+\int_{0}^{\infty} f(t) \zeta\left(1_{[0, t)} f\right) \mathrm{d} \chi_{t} \quad \text { for all } f \in L^{2}\left(\mathbb{R}_{+}\right),
$$

where $\chi$ is the normal martingale such that $\chi_{t}=\nu_{t}-t$ for all $t \in \mathbb{R}_{+}[3$, Section II.1].

Lemma A.10. In the setting of Notation $\overline{A .9}$, let $\phi$ be a bounded process on $(\Omega, \mathcal{A}, \mathbb{P})$ adapted to the Poisson filtration and let $F_{t}=U_{P} \widehat{\phi}_{t} U_{P}^{*} \in B(\mathcal{F})$ for all $t \in \mathbb{R}_{+}$, where $\widehat{\phi}_{t}$ acts as multiplication by $\phi_{t}$. Then

$$
U_{P} \int_{0}^{\infty} \phi_{t} \mathrm{~d} \chi_{t} U_{P}^{*}=\int_{0}^{\infty} F_{t} \mathrm{~d}\left(N_{t}-t\right) \quad \text { on } \mathcal{E} .
$$

Proof. For all $f \in L^{2}(\mathbb{R})$ and $t \in \mathbb{R}_{+}$, let

$$
E_{t} \zeta(f):=\zeta\left(1_{[0, t)} f\right) \quad \text { and } \quad D_{t} \zeta(f):=f(t) E_{t} \zeta(f) .
$$

Recall that the quadratic variation $[\chi]_{t}=\chi_{t}+t$ for all $t \in \mathbb{R}_{+}$. 
Thus, if $f, g \in L^{2}\left(\mathbb{R}_{+}\right)$then

$$
\begin{aligned}
& \mathbb{E}\left[\overline{\zeta(f)} \int_{0}^{\infty} \phi_{t} \mathrm{~d} \chi_{t} \zeta(g)\right] \\
& =\mathbb{E}\left[\left(1+\int_{0}^{\infty} D_{t} \overline{\zeta(f)} \mathrm{d} \chi_{t}\right) \int_{0}^{\infty} \phi_{t} \mathrm{~d} \chi_{t} \zeta(g)\right] \\
& =\mathbb{E}\left[\left(\int_{0}^{\infty} \phi_{t} \mathrm{~d} \chi_{t}+\int_{0}^{\infty} D_{t} \overline{\zeta(f)} \int_{0}^{t} \phi_{s} \mathrm{~d} \chi_{s} \mathrm{~d} \chi_{t}\right.\right. \\
& \left.\left.\quad+\int_{0}^{\infty} \int_{0}^{t} D_{s} \overline{\zeta(f)} \mathrm{d} \chi_{s} \phi_{t} \mathrm{~d} \chi_{t}+\int_{0}^{\infty} D_{t} \overline{\zeta(f)} \phi_{t} \mathrm{~d}\left(\chi_{t}+t\right)\right)\left(1+\int_{0}^{\infty} D_{t} \zeta(g) \mathrm{d} \chi_{t}\right)\right] \\
& =\int_{0}^{\infty} \mathbb{E}\left[D_{t} \overline{\zeta(f)} \phi_{t}+\phi_{t} D_{t} \zeta(g)+D_{t} \overline{\zeta(f)} \int_{0}^{t} \phi_{s} \mathrm{~d} \chi_{s} D_{t} \zeta(g)+\int_{0}^{t} D_{s} \overline{\zeta(f)} \mathrm{d} \chi_{s} \phi_{t} D_{t} \zeta(g)\right. \\
& =\int_{0}^{\infty} \mathbb{E}\left[D_{t} \overline{\zeta(f)} \phi_{t} E_{t} \zeta(g)+E_{t} \overline{\zeta(f)} \phi_{t} D_{t} \zeta(g)+D_{t} \overline{\zeta(f)} \phi_{t} \int_{0}^{t} D_{s} \zeta(g) \mathrm{d} \chi_{s}\right] \mathrm{d} s \\
& =\int_{0}^{\infty} \mathbb{E}\left[E_{t} \overline{\zeta(f)}\left(\overline{f(t)} \phi_{t}+\phi_{t} g(t)+\overline{f(t)}\left(\phi_{t}+\int_{0}^{t} \phi_{s} \mathrm{~d} \chi_{s}\right) g(t)\right) E_{t} \zeta(g)\right] \mathrm{d} t .
\end{aligned}
$$

Replacing $f, g$ and $\phi$ by $1_{[0, t]} f, 1_{[0, t]} g$ and $1_{[0, t]} \phi$, respectively, it follows that

$$
\begin{aligned}
\alpha_{t} & :=\mathbb{E}\left[\overline{\zeta\left(1_{[0, t]} f\right)} \int_{0}^{t} \phi_{s} \mathrm{~d} \chi_{s} \zeta\left(1_{[0, t]} g\right)\right] \\
& =\int_{0}^{t}(\overline{f(s)}+g(s)+\overline{f(s)} g(s)) \mathbb{E}\left[E_{s} \overline{\zeta(f)} \phi_{s} E_{s} \zeta(g)\right]+\overline{f(s)} g(s) \alpha_{s} \mathrm{~d} s,
\end{aligned}
$$

so

$$
\frac{\mathrm{d}}{\mathrm{d} t}\left(\alpha_{t} \exp \left(\int_{t}^{\infty} \overline{f(s)} g(s) \mathrm{d} s\right)\right)=(\overline{f(t)}+g(t)+\overline{f(s)} g(s)) \mathbb{E}\left[\overline{\zeta(f)} \phi_{t} \zeta(f)\right]
$$

and

$$
\mathbb{E}\left[\overline{\zeta(f)} \int_{0}^{\infty} \phi_{t} \mathrm{~d} \chi_{t} \zeta(g)\right]=\int_{0}^{\infty}(\overline{f(t)}+g(t)+\overline{f(t)} g(t)) \mathbb{E}\left[\overline{\zeta(f)} \phi_{t} \zeta(g)\right] \mathrm{d} t .
$$

\section{References}

[1] L. AcCardi \& K. Sinha, Quantum stop times, in Quantum Probability and Applications IV (L. Accardi \& W. von Waldenfels, eds.), 68-72, Lecture Notes in Math. 1396, Springer, Berlin, 1989.

[2] D. Applebaum, Stopping unitary processes in Fock space, Publ. RIMS Kyoto Univ. 24 (1988), 697-705. 
[3] S. Attal, Classical and quantum stochastic calculus, in Quantum Probability Communications X (R.L. Hudson \& J.M. Lindsay, eds.), 1-52, World Scientific, Singapore, 1998.

[4] S. Attal \& A. Coquio, Quantum stopping times and quasi-left continuity, Ann. Inst. H. Poincaré Probab. Statist. 40 (2004), no.4, 497-512.

[5] S. Attal \& J.M. Lindsay, Quantum stochastic calculus with maximal operator domains, Ann. Probab. 32 (2004), no.1A, 488-529.

[6] S. Attal \& K.R. Parthasarathy, Strong Markov processes and the Dirichlet problem in von Neumann algebras, in Stochastic analysis and applications (Powys, 1995) (I.M. Davies, A. Truman \& K.D. Elworthy, eds.), 53-75, World Scientific, Singapore, 1996.

[7] S. Attal \& K.R. Parthasarathy, Strong Markov processes and the Dirichlet problem in $C^{*}$-algebras, preprint no.357, Institut Fourier, Université Grenoble Alpes, 1996.

[8] S. Attal \& K.B. Sinha, Stopping semimartingales on Fock space, in Quantum Probability Communications X (R.L. Hudson \& J.M. Lindsay, eds.), 171-185, World Scientific, Singapore, 1998.

[9] C. Barnett \& T. Lyons, Stopping noncommutative processes, Math. Proc. Cambridge Philos. Soc. 99 (1986), no.1, 151-161.

[10] C. Barnett \& B. Thakrar Time projections in a von Neumann algebra, J. Operator Theory 18 (1987), no.1, 19-31.

[11] C. Barnett \& B. Thakrar, A noncommutative random stopping theorem, J. Funct. Anal. 88 (1990), no.2, 342-350.

[12] C. Barnett \& I.F. Wilde, Random times and time projections, Proc. Amer. Math. Soc. 110 (1990), no.2, 425-440.

[13] C. Barnett \& I. Wilde, Random times, predictable processes and stochastic integration in finite von Neumann algebras, Proc. London Math. Soc. (3) 67 (1993), no.2, 355-383.

[14] A.C.R. Belton, Quantum $\Omega$-semimartingales and stochastic evolutions, J. Funct. Anal. 187 (2001), no.1, 94-109.

[15] A.C.R. Belton, An isomorphism of quantum semimartingale algebras, Q. J. Math. 55 (2004), no.2, 135-165.

[16] A.C.R. Belton \& K.B. Sinha, Stopping the CCR flow and its isometric cocycles, Q. J. Math. 65 (2014), no.4, 1145-1164.

[17] A.C.R. Belton \& K.B. Sinha, The cocycle identity holds under stopping, Q. J. Math. 68 (2017), no.3, 817-830.

[18] B.V.R. Bhat \& K.R. Parthasarathy, Markov dilations of nonconservative dynamical semigroups and a quantum boundary theory, Ann. Inst. H. Poincaré Probab. Statist. 31 (1995), no.4, 601-651. 
[19] A. Coquio, The optional stopping theorem for quantum martingales, J. Funct. Anal. 238 (2006), no.1, 149-180.

[20] R.L. Hudson, The strong Markov property for canonical Wiener processes, J. Funct. Anal. 34 (1979), no.2, 266-281.

[21] R.L. Hudson, Stop times in Fock space quantum probability, Stochastics 79 (2007), no.34, 383-391.

[22] R.L. Hudson, Stopping Weyl processes, in Recent development in stochastic dynamics and stochastic analysis (J. Duan, S.L. Luo and C. Wang, eds.), 185-193, World Scientific, Singapore, 2010.

[23] R.L. Hudson \& K.R. Parthasarathy, Quantum Ito's formula and stochastic evolutions, Comm. Math. Phys. 93 (1984), no.3, 301-323.

[24] J.M. Lindsay, Independence for quantum stochastic integrators, in Quantum Probability E Related Topics VI (L. Accardi, ed.), 325-332, World Scientific, Singapore, 1991.

[25] J.M. Lindsay, Quantum stochastic analysis - an introduction, in Quantum Independent Increment Processes I (M. Schürmann \& U. Franz, eds.), 181-271, Lecture Notes in Math. 1865, Springer, Berlin, 2005.

[26] A. ŁuCZAK, Structure of the time projection for stopping times in von Neumann algebras, Internat. J. Theoret. Phys. 44 (2005), no.7, 909-917.

[27] P.-A. Meyer, Éléments de probabilités quantiques IV. Probabilités sur l'espace de Fock, in Séminaire de Probabilités XX (J. Azéma \& M. Yor, eds.), 249-285, Lecture Notes in Math. 1204, Springer, Berlin, 1986.

[28] P.-A. Meyer, Éléments de probabilités quantiques VIII. Temps d'arrêt sur l'espace de Fock, in Séminaire de Probabilités XXI (J Azéma, P.-A. Meyer \& M. Yor, eds.), 63-78, Lecture Notes in Math. 1247, Springer, Berlin, 1987.

[29] K.R. Parthasarathy, Quantum probability and strong quantum Markov processes, in Quantum Probability Communications XII (S. Attal \& J.M. Lindsay, eds.), 59-138, World Scientific, Singapore, 2003

[30] K.R. Parthasarathy \& K.B. Sinha, Stop times in Fock space stochastic calculus, Probab. Theory Related Fields 75 (1987), no.3, 317-349.

[31] J.-L. Sauvageot, First exit time: a theory of stopping times in quantum processes, in Quantum Probability and Applications III (L. Accardi \& W. von Waldenfels, eds.), 285-299, Lecture Notes in Math. 1303, Springer, Berlin, 1988.

[32] K.B. SinHA, Quantum stop times, in Quantum Probability Communications XII (S. Attal \& J.M. Lindsay, eds.), 195-207, World Scientific, Singapore, 2003. 